\title{
Mean-Field Equation for the Depletion Thickness
}

\author{
Gerard J . Fleer* \\ Laboratory for Physical Chemistry and Colloid Science, Wageningen University, \\ 6703 HB Wageningen, The Netherlands
}

\author{
Alexander M. Skvortsov \\ Chemical-Pharmaceutical Academy, Prof. Popova 14, 197022 St. Petersburg, Russia

\section{Remco Tuinier} \\ Forschungszentrum J ülich, Institut für F estkörperforschung, 52425 J ülich, Germany \\ Received April 22, 2003; Revised Manuscript Received J uly 30, 2003
}

\begin{abstract}
We derive a general equation for the depletion thickness $\delta$ next to a flat wall in a solution of nonadsorbing polymer, which is easily extended to spherical geometry. This equation has the simple form $\delta^{-2}=\delta_{0}{ }^{-2}+\xi^{-2}$. Here, $\delta_{0}$ is the value of $\delta$ in the limit of infinite dilution, which depends only on the chain length: $\delta_{0}=2 \mathrm{R} / \sqrt{\pi}$, where $\mathrm{R}$ is the radius of gyration of the polymer. The parameter $\xi$ is a correlation length in solution, which depends on the polymer concentration $\varphi_{\mathrm{b}}$ and the solvency $\chi_{\text {, but }}$ not on the chain length. We use a mean-field form of $\xi=\xi\left(\chi, \varphi_{b}\right)$ which provides a smooth crossover from good to $\Theta$ solvency conditions. We show that the depletion thickness is actually a generalized bulk solution correlation length which does depend on chain length. In all cases the profile for a flat wall is given by $\rho=\varphi / \varphi_{\mathrm{b}}=\tanh ^{2}(\mathrm{z} / \delta)$. The extension to a sphere of radius a is also very simple: $\rho_{\mathrm{s}}=[\mathrm{z} / \mathrm{a}+\tanh (\mathrm{z} / \delta)]^{2} /$ $[z / a+1]^{2}$. These analytical results are compared to numerical self-consistent-field computations, whereby the segment-wall repulsion in the lattice model is chosen in accordance with the boundary condition $\rho(0)=0$ in the continuum model. The agreement is nearly perfect for good solvents and large particles. For a $\Theta$ solvent our simple analytical model overestimates $\delta$ slightly; in this case the $\tanh ^{2}$ profile is not strictly valid, and we derive a corrected analytical form. For smaller particles, also a slight overestimation of the width of the depletion zone is found. However, in all cases the trends are predicted very well. Our simple equations allow, in principle, analytical expressions for the surface tension, for the distribution coefficient in size-exclusion chromatography, and for interaction potentials and phase diagrams of colloids with nonadsorbing polymer.
\end{abstract}

\section{Introduction}

Dispersions of colloidal particles mixed with nonadsorbing polymer chains are interesting both from a physics point of view and practically. Physically, such a system may be considered as a colloidal liquid in which the colloidal particles interact through some effective potential, which is determined by the concentration and size of the nonadsorbing polymer chains. ${ }^{1}$ These dispersions further also reflect practical situations in biological systems and industrial products such as proteinpolysaccharide mixtures in food ${ }^{2}$ or latex-polymer mixtures in paint. ${ }^{3}$ The depletion of nonadsorbing polymer has also very important applications in sizeexclusion chromatography, which is widely used in polymer analysis. ${ }^{4}$

Asakura and Oosawa ${ }^{5}$ were the first to give a theoretical explanation for this depletion and the ensuing attraction between colloidal particles due to nonadsorbing polymers. They calculated the attractive force between two parallel flat plates immersed in a solution of ideal (ghost) polymer chains. From their analysis it follows that the range of attraction is $4 R / \sqrt{\pi}$, where $R$ is the radius of gyration of the polymer. The depletion layer in which the polymer chains are effectively absent has thus a thickness $2 \mathrm{R} / \sqrt{\pi} \approx 1.13 \mathrm{R}$ on each plate.

To develop a simple description for mixtures of coll oidal spheres and (nonadsorbing) polymer, Asakura and Oosawa 6 and later Vrij $^{7,8}$ proposed to replace the polymer chains by penetrable hard spheres (PHS's) with radius R. PHS's are hard spheres for the colloidal particles but fully penetrable for other PHS's. This coarse-grained approach provided extensions that allow a prediction of the phase behavior of polymer (PHS)colloid mixtures, $, 9,10$ which for small size ratios R/a (where $a$ is the sphere radius) accurately predicts the experimental binodal. ${ }^{11}$

For larger size ratios, however, the PHS/sphere approximation for polymer-colloid mixtures predicts a binodal at too small polymer concentrations ${ }^{11}$ for two main reasons. First, the depletion thickness decreases to values that are significantly smaller than $\mathrm{R}$ with increasing size ratio R/a. ${ }^{12-15}$ This effect of the wrapping of a polymer chain around a sphere makes the attraction between the spheres weaker. Second, at concentrations beyond coil overlap the depletion thickness at a flat plate becomes smaller than $2 R / \sqrt{\pi}$ because of the increasing osmotic pressure. This compression of the depletion layer occurs for polymer chains in a $\Theta$ solvent and is even stronger for excluded-volume chains. In semidilute solutions the characteristic length scale becomes the correlation length (blob size) $\xi$, which is independent of the chain length, as already advanced by J oanny et al. ${ }^{16}$

To take into account the interactions between the polymer segments and their effects on the polymer density profile and depletion potential, several types of approaches have been developed, such as mean-field theory, ${ }^{17}$ polymer-colloid liquid-state theory, ${ }^{18}$ the product-function approximation, ${ }^{19}$ a Gaussian-core model, ${ }^{20}$ 
field theories, ${ }^{21,22}$ and computer simulations. ${ }^{20,23,24}$ Predictions of the phase diagram were made using polymercolloid liquid-state theory, ${ }^{18}$ free-volume theory, ${ }^{25}$ a Gaussian-core model, ${ }^{26}$ and density functional theory. 27,28 All these theories consider the limiting case of good solvency conditions (full excluded-volume limit). The original theory, giving a depletion thickness $2 R / \sqrt{\pi}, 5$ is valid in the other limit of ideal (i.e., noninteracting) polymer chains.

So far, there is no simple theory available that predicts the depletion thickness, the colloid-colloid interaction, and the phase behavior for arbitrary concentration and solvent quality. The solvent quality is classically described using the Flory-Huggins parameter $\chi$, which is related to the excluded volume per segment $v$ as $v=1-2 \chi$. In the full excluded-volume limit $(\chi=0)$ there are only athermal interactions, whereas in a $\Theta$ solvent $(\chi=0.5)$ the excluded volume as defined by $v=1-2 \chi$ vanishes; higher-order concentration terms then become important. In practice, however, the entire range of solvencies and especially the range for $\chi$ in between 0.3 and 0.5 are rel evant. Even bad solvents $(\chi>0.5)$ are of practical interest.

There is thus a need for a theory that can be used to describe polymer-colloid mixtures for arbitrary concentration and solvent quality. Numerical self-consistentfield (SCF) theory has been developed that allows to compute, on a mean-field level, the density profiles and depletion thickness for a wide variety of conditions. ${ }^{29-32}$ To recognize the trends in the various quantities and to estimate other physical properties, such as the surface tension, 33,34 the wall-polymer potential, 20 the polymer-mediated colloid-colloid interaction, 35,36 and the polymer - colloid phase behavior, ${ }^{25}$ it is desirable to have analytical expressions. These are available for ideal chains in the limit of infinite dilution and for semidilute good solvents. In this semidilute regime a simple tanh ${ }^{2}$ profile was derived for the relative polymer density profile $\rho(\mathrm{z})=\varphi(\mathrm{z}) / \varphi_{\mathrm{b}}$ near a flat wall, ${ }^{37,38}$ using the ground-state approximation. ${ }^{38-41}$ Odijk $^{42}$ calculated the polymer density profilenear an infinitesimally small sphere in a semidilute polymer solution. The large- and small-sphere semi dilute limits were recently combined to an equation which predicts the polymer density profile for an arbitrary sphere radius. ${ }^{43}$

In this paper we present a general analytical meanfield equation which describes the depletion thickness and polymer density profile for arbitrary solvent quality $\chi$, chain length $\mathrm{N}$, polymer bulk concentration $\varphi_{\mathrm{b}}$, and polymer-colloid size ratio R/a remarkably well. The main ingredients are the following: (i) The known analytical results for the dilute limit, which are complicated as they contain (complementary) error functions, are rewritten in an approximate form $\rho=\tanh ^{2}(z)$ $\left.\delta_{0}\right)$, where $\delta_{0}=\delta_{0}(R)$ equals $2 R / \sqrt{\pi}$. (ii) The wellknown expression $\rho=\tanh ^{2}(z / \xi)$ for the semidilute limit is generalized by using a correlation length $\xi=\xi\left(\chi, \varphi_{\mathrm{b}}\right)$ which ensures a smooth crossover from good solvents to $\Theta$ conditions (and slightly beyond). (iii) The connection between the two limits is made by writing $\rho=$ $\tanh ^{2}(z / \delta)$, where the depletion thickness $\delta=\delta\left(\mathrm{R}, \chi, \varphi_{\mathrm{b}}\right)$ is a chain-length-dependent correlation length, given by the extremely simple relation $\delta^{-2}=\delta_{0}{ }^{-2}+\xi^{-2}$. (iv) The generalization to spheres is made in a similar way as recently proposed by Tuinier and Lekkerkerker. ${ }^{43}$

The analytical results are compared to numerical SCF data. In this comparison, attention is paid to the correct mapping of continuum and lattice models: the usual boundary condition $\rho(0)=0$ translates into a certain (weak) segment/wall repulsion in the lattice model.

The outline of the paper is as follows. In section 2 we start from the Edwards equation (which is the continuum analogue of the recurrence relation used in a lattice). We introduce the segment potential $u_{b}$ in the bulk solution (with respect to infinite dilution) in a way that later (section 5) allows to define $\xi\left(\chi, \varphi_{b}\right)$ for any solvency and concentration. We further define the depl etion thickness as the zeroth moment of the profile. In section 3 we review some known expressions for the polymer density profile and the depletion thickness in the limit of ideal chains at vanishing polymer concentration, and we rewrite those in an approximate tanh ${ }^{2}$ form. In section 4 we recall the basics of the groundstate approximation (GSA), needed to find approximate solutions of the Edwards equation for finite concentrations. We apply GSA in section 5 to semidilute solutions, both for good solvents and for $\Theta$ conditions; for the latter case some new results are presented. In section 6 we derive a general expression for the depletion thickness at arbitrary solvent qual ity and polymer concentration, using GSA for a slit. In section 7 we describe the extension to spheres. Finally, we present and discuss various analytical results and compare them with numerical SCF results in section 8. The appendix gives some information about how to properly match continuum and lattice models.

\section{Basic Equations}

Polymer conformations near an interface may be described by discrete or continuum models. The continuum description is based upon the Edwards equation ${ }^{38-41}$ for the partition function $G(z, s)$, which gives the statistical weight that walks of (contour) length $\mathrm{S}$ in a potential field $u(z)$ end at position $z$. The governing equation may be formulated as

$$
\frac{1}{6} \nabla^{2} G=\frac{\partial G}{\partial s}+\left(u-u_{b}\right) G
$$

where $\nabla^{2}$ is the Laplace operator and where the spatial coordinate $z$ occurring in $\nabla^{2}$ is in units of the bond length. We have chosen a form that ensures that $u-$ $u_{b}$ is zero in the homogeneous bulk solution. Both $u=$ $\mathrm{u}(\mathrm{z})$ and $\mathrm{u}_{\mathrm{b}}=\mathrm{u}(\infty)$ are in units $\mathrm{kT}$ and defined with respect to infinite dilution. In the general case, $u(z)$ may contain an adsorption energy contribution, ${ }^{31}$ but we restrict ourselves to the case of depletion where the solid surface is energetically neutral or repulsive; then $u-$ $u_{b}$ is negative in the surface region, and the usual boundary condition for eq 1 is $\mathrm{G}(0, \mathrm{~s})=0$ at the surface $(z=0)$. In this way, the segment/surface repulsion does not enter explicitly. In discrete descriptions like the Scheutjens-Fleer layer model, ${ }^{29-31}$ eq 1 is replaced by a recurrence relation, with a boundary condition specifying the segment/surface interaction.

In a mean-field picture the potential $u(z)$ can be described as a Flory-Huggins field: : $31,44-47^{-1}$

$$
\begin{gathered}
\mathrm{u}(\mathrm{z})=-\ln [1-\varphi(\mathrm{z})]-2 \chi \varphi(\mathrm{z}) \\
\mathrm{u}_{\mathrm{b}}=-\ln \left(1-\varphi_{\mathrm{b}}\right)-2 \chi \varphi_{\mathrm{b}}
\end{gathered}
$$

where $\chi$ is the Flory-Huggins solvency parameter, $\varphi(z)$ is the local polymer concentration, and $\varphi_{\mathrm{b}}=\varphi(\infty)$ is the volume fraction of polymer segments in the bulk solution. 
In the form of eq 2a, nonlocal energy effects arising from the fact that in the depletion layer the number of contacts with "inward" segments (at lower $\mathrm{z}$ ) is different from that with "outward" segments (higher z) are neglected (see eq 20 in section 5.3 for a correction). In a common approximation for good solvents, ${ }^{37,38}$ eq 2 is expanded up to the linear term, giving $\mathrm{u} \approx \mathrm{v} \varphi$ and $\mathrm{u}_{\mathrm{b}} \approx$ $\vee \varphi_{\mathrm{b}}$, where $\mathrm{v} \equiv 1-2 \chi$ is the $\mathrm{E}$ dwards excluded-volume parameter.

With the logarithmic form of eq 2, the solution of eq 1 cannot be obtained analytically, and a numerical procedure is required to compute $G(z, s)$. To find this solution, the concentrations occurring in eq 2 have to be expressed as a function of $G(z, s)$. The relative concentration $\rho_{\mathrm{e}}(\mathrm{z})$ of end points and that of all chain segments $\rho(z)$ are given by

$$
\begin{gathered}
\rho_{\mathrm{e}} \equiv \frac{\mathrm{N} \varphi_{\mathrm{e}}(\mathrm{z})}{\varphi_{\mathrm{b}}}=\mathrm{G}(\mathrm{z}, \mathrm{N}) \\
\rho \equiv \frac{\varphi(\mathrm{z})}{\varphi_{\mathrm{b}}}=\frac{1}{\mathrm{~N}} \int_{0}^{\mathrm{N}} \mathrm{G}(\mathrm{z}, \mathrm{s}) \mathrm{G}(\mathrm{z}, \mathrm{N}-\mathrm{s}) \mathrm{ds}
\end{gathered}
$$

Both $\rho_{\mathrm{e}}(\mathrm{z})$ and $\rho(\mathrm{z})$ are defined such that they are unity in the bulk solution, where $\mathrm{G}(\infty, \mathrm{s})=1$.

From either $\rho_{\mathrm{e}}(\mathrm{z})$ or $\rho(\mathrm{z})$ the depletion thickness $\delta$ may be obtained. It is defined as the distance from the surface at which a step profile $\varphi(z)=\Phi(z-\delta) \varphi_{\mathrm{b}}$, where $\Phi(\mathrm{x})$ is the Heaviside step function, would give the same negative adsorption as the continuous profile. For a flat wall we have for this negative adsorption $\theta^{\mathrm{ex}}=-\varphi_{\mathrm{b}} \delta$, where $\theta^{\mathrm{ex}}=\Gamma / \mathrm{A}$ is the negative adsorption per unit area. In a lattice model, $\theta$ ex is expressed per lattice site. On the basis of this definition, we find

$$
\delta=-\frac{\theta^{\mathrm{ex}}}{\varphi_{\mathrm{b}}}=\int_{0}^{\infty}(1-\rho) \mathrm{dz}=\int_{0}^{\infty}\left(1-\rho_{\mathrm{e}}\right) \mathrm{dz}
$$

The depletion thickness thus corresponds to the zeroth moment of the concentration profile. The generalization of eq 5 to spherical geometry will be given in section 7.4 (eq 33).

\section{Exact Expressions for $\varphi_{b} \rightarrow 0$ in Flat Geometry}

Exact analytical solutions of eq 1 are only known for the limiting case $u(z)=0$ and $u_{b}=0$, corresponding to extremely low bulk concentrations $\varphi_{\mathrm{b}}$. In this section, we give two such sol utions for ideal chains (no excluded volume) at a flat wall. The extension to spheres is described in section 7.1.

3.1. Polymer near a Wall. The first case is the depletion profile next to a flat wall. Clearly, in the dilute limit the depletion thickness is only a function of the chain length $N=6 R^{2}$ (where $R$ is in units of the bond length). The solutions are most easily expressed by measuring the distance $z$ from the wall in units of the gyration radius $\mathrm{R}$ of the polymer, using the parameter $\xi \equiv \mathrm{z} / 2 \mathrm{R}$. For a flat plate, Eisenriegler ${ }^{48,49}$ derived expressions for $\rho_{\mathrm{e}}(\mathrm{z})$ and $\rho(\mathrm{z})$. With the boundary condition $\mathrm{G}(0, \mathrm{~s})=0$ they are

$$
\begin{gathered}
\rho_{\mathrm{e}}=\operatorname{erf} \xi \\
\rho=2 \psi(\xi)-\psi(2 \zeta)
\end{gathered}
$$

where the function $\psi(\mathbf{x})$ is defined as

$$
\psi(x) \equiv 1-4 i^{2} \operatorname{erfc} x=\operatorname{erf} x+\frac{2 x}{\sqrt{\pi}} e^{-x^{2}}-2 x^{2} \operatorname{erfc} x
$$

The function $\mathrm{i}^{2}$ erfc $\mathrm{x}$ is the second iterated complementary error function. A plot of the functions $\psi(\mathbf{x})$ and $\rho(\mathbf{x})$ is given in Figure 1 (solid curves).

Application of either version of eq 5 gives with eq 6 (or another polymer density such as the center-ofmass ${ }^{50}$ ) for the depletion layer thickness $\delta_{0}$ in this case

$$
\delta_{0}=\frac{2}{\sqrt{\pi}} \mathrm{R}
$$

which corresponds to the result of Asakura and Oosawa. ${ }^{5}$ We use the index 0 in $\delta_{0}$ to denote the limit of zero concentration. We note that an equation of the type of eq $8\left(\delta_{0}=1.13 R\right)$ does also apply to excluded-volume chains $\left(R \sim N^{0.6}\right)$ at infinite dilution: Hanke et al. ${ }^{21}$ found $\delta_{0}=1.07 \mathrm{R}$ in this case.

Figure 2 gives a plot of the end-point distribution $\rho_{\mathrm{e}}$ according to eq $6 a$ (dashed curve), the overall distribution $\rho$ according to eq $6 \mathrm{~b}$ (solid), and the distribution $\rho_{\mathrm{m}}$ of the middle segment (dotted). The latter is defined as $\rho_{\mathrm{m}} \equiv \mathrm{N} \varphi_{\mathrm{m}} / \varphi_{\mathrm{b}}$. F or an arbitrary segment $\mathrm{s}, \rho(\mathrm{s})$ is given as $\operatorname{erf}(z \sqrt{6} / 2 \sqrt{s}) \operatorname{erf}(z \sqrt{6} / 2 \sqrt{\mathrm{N}-\mathrm{s}})$; for the middle segment (with $\mathrm{s}=\mathrm{N} / 2$ ) we have $\rho_{\mathrm{m}}=\operatorname{erf}^{2}(\xi \sqrt{2}$ ).

All three curves have the same integral; the scaled distance $\xi_{0}=\delta_{0} / 2 \mathrm{R}=1 / \sqrt{\pi}$ gives equal areas for the parts under the curves for $\xi<1 / \sqrt{\pi}$ and those above the curves for $\xi>1 / \sqrt{\pi}$. For $z<\delta_{0}$ the chain ends are overrepresented as compared to the average of all segments; for $z>\delta_{0}$ the opposite is the case. It is also clear that the overall profile is rather close to that of the middle segment: $\rho$ is between $\rho_{\mathrm{e}}$ and $\rho_{\mathrm{m}}$ but much closer to $\rho_{\mathrm{m}}$. Hence, if one would need a simple approximation for the rather complicated expression $6 \mathrm{~b}$, one could replace $\rho$ by $\rho_{\mathrm{m}}$.

However, an even better approximation is possible: it turns out that the function $\rho(x)=2 \psi(x)-$ $\psi(2 \mathrm{x})$ for a flat wall is very accurately described as the square of a hyperbolic tangent: $\rho(x) \approx \tanh ^{2}(x \sqrt{\pi})$; see Figure 1 (dotted curve). This was also noted by Tuinier et al. ${ }^{14}$ The integral of eq 5 with this approximate form of $\rho(\xi)$ gives exactly the correct depletion thickness $\delta_{0}=(2 / \sqrt{\pi}) \mathrm{R}$. Hence, we will approximate $\rho(\mathrm{z})$ as $\tanh ^{2}(z \sqrt{\pi} / 2 R)$ or, with eq 8

$$
\rho=\tanh ^{2}\left(\frac{\mathbf{z}}{\delta_{0}}\right)
$$

For the case of a sphere (section 7) we need also an approximation for the function $\psi(\mathrm{x})$. This function is well approximated by $\tanh (x \sqrt{\pi})$, as also shown in Figure 1 . The agreement is slightly less than for $\rho=\tanh ^{2}(x \sqrt{\pi})$ but still rather good.

3.2. Polymer in a Slit. The second case which is relevant for our present purpose and for which an exact solution is available is that of ideal polymer chains in a slit of width $D$ which is in equilibrium with a very dilute bulk solution. This case was first treated by Casassa ${ }^{51}$ in the context of size exclusion chromatography (SEC). We do not give the general solution for the concentration profile in the slit (although we do consider the profile in the limit of narrow slits). For the general case, we restrict ourselves to the average value $\bar{\rho}=\bar{\varphi} / \varphi_{\mathrm{b}}$, where 


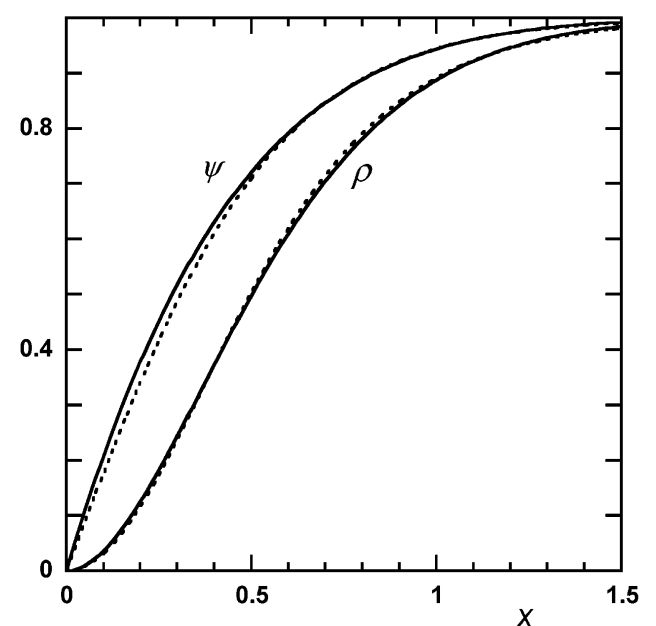

Figure 1. Functions $\psi(x)$ and $\rho(x)=2 \psi(x)-\psi(2 x)$. The solid curves are the exact expressions eq 7 and eq 6b, respectively; the dotted curves are the approximations $\tanh (x \sqrt{\pi})$ and $\tanh ^{2}(x \sqrt{\pi})$.

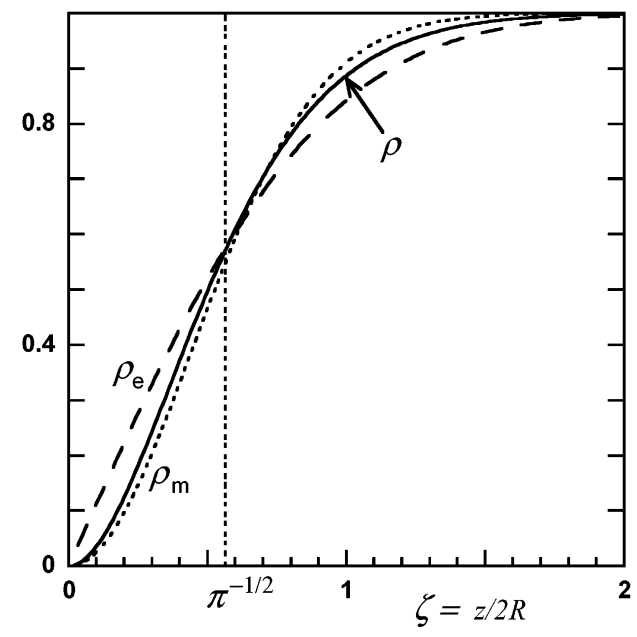

Figure 2. Distribution of end segments $\rho_{\mathrm{e}}$ (eq $6 \mathrm{a}$, dashed curve), the overall distribution $\rho$ (eq $6 \mathrm{~b}$, solid), and the distribution $\rho_{m}=\operatorname{erf}^{2}(\zeta \sqrt{2})$ of the middle segment (dotted) as a function of the scaled distance $\xi=\mathrm{z} / 2 \mathrm{R}$ from the surface, in the limit of infinite dilution. The dotted vertical line corresponds to $\zeta_{0}=\delta_{0} / 2 \mathrm{R}=1 / \sqrt{\pi}$, where $\delta_{0}$ is the depletion thickness, given by eq 8 .

$\bar{\varphi}$ is the average concentration in the slit and $\varphi_{\mathrm{b}}$ the outside concentration. In fact, $\bar{\rho}$ is the partition coefficient for distributing the polymer over slitlike pores and a bulk solution. Assuming that $\varphi_{\mathrm{b}}$ is so low that the field terms $u$ and $u_{b}$ in eq 1 vanish and using the boundary conditions $\mathrm{G}(0, \mathrm{~s})=0$ and $\mathrm{G}(\mathrm{D}, \mathrm{s})=0$, Casas$\mathrm{sa}^{51}$ derived the following expression

$$
\bar{\rho}=\frac{8}{\pi^{2}} \sum_{n=1,3 \ldots n^{2}} \frac{1}{n^{2}} \mathrm{e}^{-(\mathrm{n} \pi \mathrm{R} / \mathrm{D})^{2}}
$$

where $D$ is the slit width and $R$ the radius of gyration. The partition coefficient depends only on the ratio R/D (or $\delta_{0} / \mathrm{D}$ ).

A plot of $\bar{\rho}$ as a function of the ratio $\delta_{0} / D=(2 / \sqrt{\pi}) \mathrm{R} / \mathrm{D}$ is given in Figure 3 (solid curve). For $\bar{\rho} \leq 0.5, \delta_{0} / \mathrm{D} \gtrsim$ 0.25 we have the narrow-pore regime, for which the first term of the summation in eq 10 suffices. Casassa showed that in this limit the concentration profile in the slit $(0<z<D)$ is a squared sine. When we replace

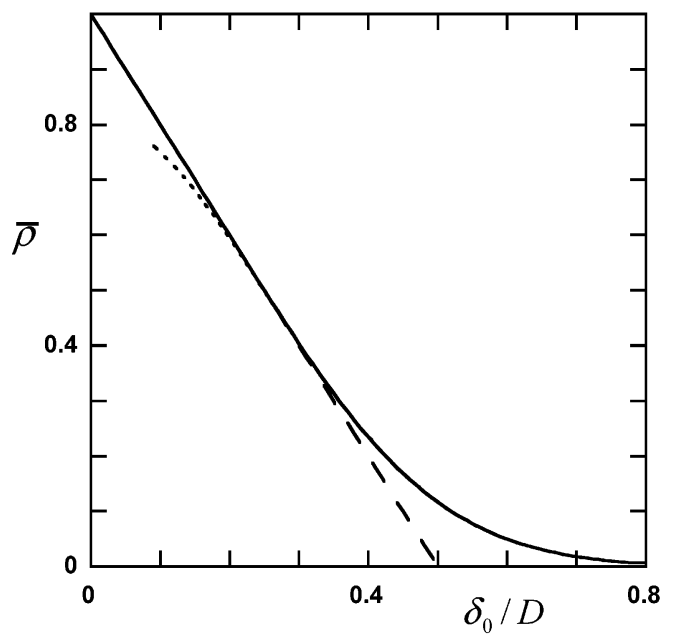

Figure 3. Distribution coefficient $\bar{\rho}=\bar{\varphi} / \varphi_{\mathrm{b}}$ for a slitlike pore of width $\mathrm{D}$ as a function of the ratio $\delta_{0} / \mathrm{D}$ in the limit of infinite dilution. The solid curve is the full result eq 10, the dotted curve is the narrow-pore limit eq $11 \mathrm{a}$, and the dashed line is the wide-pore limit eq 12.

$R$ in eq 10 by $(\sqrt{\pi} / 2) \delta_{0}$, we may write

$$
\begin{gathered}
\bar{\rho}=\frac{8}{\pi^{2}} \mathrm{e}^{-\pi^{3}\left(\delta_{0} / 2 \mathrm{D}\right)^{2}} \\
\rho=2 \bar{\rho} \sin ^{2}\left(\frac{\pi \mathrm{Z}}{\mathrm{D}}\right)
\end{gathered}
$$

This dependence according to eq 11 a is indicated in Figure 3 as the dotted curve; it is quite accurate for $\delta_{0} / D$ $\approx 0.25$.

For wide pores $\left(\delta_{0} / \mathrm{D} \lesssim 0.25\right)$ the summation in eq 10 converges to the simple expression

$$
\bar{\rho}=1-2 \delta_{0} / D
$$

This linear dependence corresponds to the dashed line in Figure 3. In this case the profile in each half of the slit is the same as that at an isolated wall and is described by eq 6 , which may be approximated as $\rho=$ $\tanh ^{2}\left(z / \delta_{0}\right)$ according to eq 9 .

\section{Ground-State Approximation (GSA)}

When $u \neq u_{b}$ in eq 1 , no exact analytical solutions are known. One can then try to find approximate solutions by using only the first term of an exact eigenvalue expansion of $\mathrm{G}(\mathrm{z}, \mathrm{s})$ :

$$
\mathrm{G}(\mathrm{z}, \mathrm{s})=\mathrm{g}(\mathrm{z}) \mathrm{e}^{\epsilon \mathrm{s}}
$$

where $e$ is the largest (ground-state) eigenvalue and $g(z)$ the ground-state eigenfunction. In eq 13, the $z$ and $s$ dependencies are now separated. Upon substitution of eq 13 into eq 1, an ordinary differential equation in $\mathrm{g}$ results:

$$
\nabla^{2} g=6\left(\epsilon+u-u_{b}\right) g
$$

With the logarithmic form of $u$ as given in eq 2 , this equation still cannot be solved analytically. Usually, an expanded form of $u$ is chosen; we return to this point in the next section.

Upon substitution of eq 13 into eqs 3 and 4, it turns out that $\rho_{\mathrm{e}}$ is proportional to $\mathrm{g}$ and $\rho$ to $\mathrm{g}^{2}$ : 


$$
\begin{aligned}
& \rho_{\mathrm{e}}=\mathrm{e}^{\epsilon \mathrm{N}} \mathrm{g} \\
& \rho=\mathrm{e}^{\epsilon \mathrm{N}} \mathrm{g}^{2}
\end{aligned}
$$

We will use eqs 13-15 for two cases: in section 5 we consider depletion next to a flat wall in a semidilute solution (both in good solvents and under $\Theta$ conditions), and in section 6 we treat a narrow slit. In the former case (semidilute solution at a single surface) $\epsilon$ is zero because in the bulk solution $\left(u=u_{b}\right) \nabla^{2} g$ is zero; then $\rho=\mathrm{g}^{2}$ according to eq $15 \mathrm{~b}$ so that $\mathrm{g}=1$ in the bulk solution. In the second case (section 6) we use GSA to extend Casassa's equation for a narrow slit to finite external concentrations; in that case $\epsilon$ is negative, and its value is determined by the slit width $D$, the bulk concentration $\varphi_{\mathrm{b}}$, and the solvency $\chi$.

In this paper, we restrict ourselves to the case of depletion, where the segment/wall interaction is repulsive so that the concentration close to the surface is small. However, as shown by Fleer et al., 52 GSA can also be successfully applied to the case of attractive interactions (polymer adsorption); in this case the surface concentration is high, and the resulting equations are necessarily more complicated.

\section{GSA in Semidilute Solutions}

5.1. The Correlation Length in Semidilute Solutions. In semidilute solutions (beyond coil overlap) the chain-length dependence disappears, and the appropriate length scale is no longer the gyration radius $\mathrm{R}$ but the blob size $\xi$ which depends only on concentration (and solvency). De Gennes 37,38 expanded eq 2 in terms of $\varphi$ and retained only the linear term: $\mathrm{u}-\mathrm{u}_{\mathrm{b}} \approx \mathrm{v}\left(\varphi-\varphi_{\mathrm{b}}\right) \approx$ $\mathrm{u}_{\mathrm{b}}(\rho-1)$, where $\mathrm{v}=1-2 \chi$ is the Edwards excludedvolume parameter and where $\mathrm{u}_{\mathrm{b}} \approx \mathrm{v} \varphi_{\mathrm{b}}$. De Gennes defined the correl ation length $\xi$ in semidilute solutions through $\xi^{-2}=3 \mathrm{v} \varphi_{\mathrm{b}}=3 \mathrm{u}_{\mathrm{b}}$. When we use the more general eq $2 b$ for $u_{b}$, we may extend this to

$$
\xi^{-2}=3 u_{b}=-3\left[\ln \left(1-\varphi_{b}\right)+2 \chi \varphi_{b}\right]
$$

Clearly, for the conditions used by De Gennes, eq 16 reduces to the familiar form $\xi^{-2}=3 \mathrm{v} \varphi_{\mathrm{b}}$.

However, eq 16 may also be used for more concentrated solutions where higher-order concentration terms contribute and even for a $\Theta$ solvent where the linear term in the expansion vanishes. Moreover, it allows for a smooth crossover between good solvents and a $\Theta$ solvent (and slightly beyond) when the solvency parameter $\chi$ is varied. Throughout this paper, we will use a correlation length $\xi$ as defined by eq 16 . We note that the prefactor used in eq 16 gives a correlation length which is twice the exponential decay length $\xi_{\text {e }}$ of the profile at large distance from the surface: $\xi=2 \xi_{\mathrm{e}}$. This enables us to use $\tanh ^{2}(\mathrm{z} / \xi)$ in the profile (see eq 18) in the same way as De Gennes, ${ }^{37}$ whereas other wise tanh ${ }^{2}$ $\left(\mathrm{z} / 2 \xi_{\mathrm{e}}\right)$ would be needed. ${ }^{32,53}$ We could call $\xi$ the blob diameter and $\xi_{\mathrm{e}}$ the blob radius.

Figure 4 gives a double-logarithmic plot of $\xi\left(\varphi_{\mathrm{b}}\right)$ (top) and of $\xi^{-2}\left(\varphi_{\mathrm{b}}\right)$ (bottom) for $\chi=0, \chi=0.4$, and $\chi=0.5$. The asymptotic behavior $\xi^{-2}=3 \mathrm{v} \varphi_{\mathrm{b}}(\chi=0,0.4)$ and $\xi^{-2}$ $=(3 / 2) \varphi_{\mathrm{b}}{ }^{2}(\chi=0.5)$ is indicated as the dotted lines. For $\chi=0$ and 0.5 the asymptotes describe $\xi$ well up to $\varphi_{\mathrm{b}} \approx$ 0.1 ; for $\chi=0.4$ this is the case up to $\varphi_{\mathrm{b}} \approx 0.02$.

For $\chi>0.5, \xi$ can only be defined through eq 16 for $\mathrm{u}_{\mathrm{b}}>0$. At a given concentration, the maximum value $\chi_{\mathrm{m}}$ foll ows from $\ln \left(1-\varphi_{\mathrm{b}}\right)+2 \chi_{\mathrm{m}} \varphi_{\mathrm{b}}=0$. This value $\chi_{\mathrm{m}}$ is

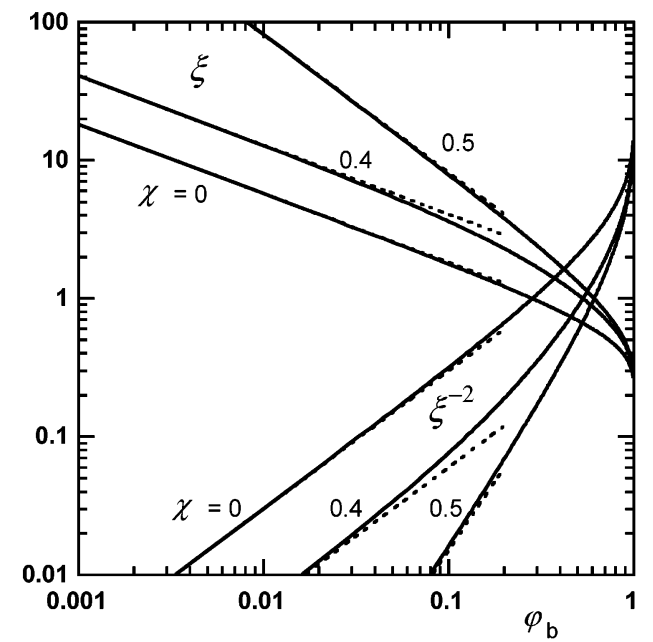

Figure 4. Correlation length $\xi$ (top) according to eq 16 and its inverse square $\xi^{-2}$ (bottom) as a function of the solution concentration $\varphi_{\mathrm{b}}$ for three solvencies, as indi cated. The dotted lines correspond to the asymptotic behavior $\xi^{-2}=3 \mathrm{v} \varphi_{\mathrm{b}}(\chi=0$, $0.4)$ and $\xi^{-2}=(3 / 2) \varphi_{b}^{2}(\chi=0.5)$.

close to the $\chi$ value corresponding to the binodal for infinite chain length (see also the discussion of Figure 9 in section 8.3).

5.2. Good Solvents. When $\epsilon=0$, eq $15 \mathrm{~b}$ reduces to $\rho=\mathrm{g}^{2}$, and up to first order in polymer concentration, $\mathrm{u}-\mathrm{u}_{\mathrm{b}}$ in eq 14 may be written as $\mathrm{u}-\mathrm{u}_{\mathrm{b}} \approx \mathrm{u}_{\mathrm{b}}(\rho-1)=$ $u_{b}\left(g^{2}-1\right)$, where $u_{b} \approx v \varphi_{b} \approx \xi^{-2 / 3}$. Then eq 14 is simplified to

$$
\frac{\xi^{2}}{2} g^{\prime \prime}=g^{3}-g
$$

where $g^{\prime \prime}$ is an abbreviation for $d^{2} g / d z^{2}$. De Gennes solved this equation by multiplying both sides by $g^{\prime \prime}=$ $\mathrm{dg} / \mathrm{dz}$ and integrating to give $\mathrm{dg} / \mathrm{d}(\mathrm{z} / \xi)=1-\mathrm{g}^{2}$. This equation is easily integrated and leads, with the boundary condition $\mathrm{g}(0)=0$, to $\mathrm{z} / \xi=$ atanh $\mathrm{g}$. Inversion is no problem:

$$
\begin{gathered}
g=\tanh \frac{z}{\xi} \\
\rho=\tanh ^{2} \frac{z}{\xi}
\end{gathered}
$$

Inserting eq 18b into eq 5 gives the depletion thickness in semidilute good solvents:

$$
\delta=\xi
$$

The depletion thickness is thus equal to the correlation length (blob diameter) $\xi$, which in semi dilute solutions is independent of the chain length. We will extend this statement to include the chain-length dependence for the general case in section 6.3.

In passing, we note that substitution of $\rho_{\mathrm{e}}=\mathrm{g}$ into eq 5 leads to a different result: $\delta=\xi \ln 2$. This shows that GSA provides only an approximate solution since both forms should give the same result when exact expressions for $\rho$ and $\rho_{\mathrm{e}}$ are available. In that case the integrals over $\rho=\mathrm{g}^{2}$ and over $\rho_{\mathrm{e}}=\mathrm{g}$ should be equal (see section 6.1 ), which is not the case for $g$ as given by eq 18a. Since GSA is expected to be more appropriate for "average segments" than for end segments, we stick to the simple form given in eq 19 . 
Another point worth noting is the use of our generalized $\xi$ in eq 17 . Strictly speaking, eq 17 is only valid with the expanded form $\xi^{-2}=3 \mathrm{v} \varphi_{\mathrm{b}}$. Yet, in eq 18 we use the generalized form of eq 16 , which is mathematically inconsistent. Our only justification is that we obtain an equation that covers a wide range of $\varphi_{\mathrm{b}}$ and $\chi$ with smooth crossovers and that, as we will show in the Results section (Figure 8), the outcome for the depletion thickness compares very favorably with the results of the Scheutjens-Fleer (SF) model, in which all eigenfunctions are taken into account. This makes our procedure, though mathematically not strict, neverthel ess useful.

5.3. $\Theta$ Solvent. Under $\Theta$ conditions the linear term in the expansion of $u$ vanishes. For the "local" form of eq 2 , the difference $u-u_{b}$ may, up to second order in polymer concentration, now be written as $\mathrm{u}-\mathrm{u}_{\mathrm{b}} \approx$ $\mathrm{u}_{\mathrm{b}}\left(\rho^{2}-1\right)=\mathrm{u}_{\mathrm{b}}\left(\mathrm{g}^{4}-1\right)$, where $\mathrm{u}_{\mathrm{b}} \approx \varphi_{\mathrm{b}}{ }^{2} / 2 \approx \xi^{-2} / 3$. This leads to eq 17 with $\mathrm{g}^{3}$ replaced by $\mathrm{g}^{5}$. The different concentration dependence of $\xi$ is automatically accounted for when the general form of eq 16 is used (which implies the same mathematical inconsistency as for a good solvent, as discussed at the end of the previous section).

However, in a $\Theta$ solvent the nonlocal effect, not accounted for in eq 2 , is relatively more important. This was al so noted by F leer et al. ${ }^{52}$ for the case of adsorbing polymer. In a more general form of eq $2 a$ the term $2 \chi \varphi$ in a concentration gradient has to be replaced by $2 \chi\langle\varphi\rangle$, where $\langle\varphi\rangle$ is the site fraction or contact fraction. In a discrete layer model like that of Scheutjens and Fleer ${ }^{29-31}\langle\varphi\rangle$ is defined as $\langle\varphi\rangle=[\varphi(z-1)+4 \varphi(z)+$ $\varphi(z+1)] / 6$. The continuum version of this expression is $\langle\varphi\rangle=\varphi+\varphi^{\prime \prime} / 6$, where $\varphi^{\prime \prime}$ is again $\mathrm{d}^{2} \varphi / \mathrm{dz}^{2}$. E quation $2 \mathrm{a}$ is now extended to

$$
\mathrm{u}=-\ln (1-\varphi)-2 \chi\left(\varphi+\varphi^{\prime \prime} / 6\right) \approx \mathrm{v} \varphi+\varphi^{2} / 2-\chi \varphi^{\prime \prime} / 3
$$

Clearly, the nonlocal term vanishes for $\chi=0, v=1$. For $\chi>0$ this nonlocal contribution is nonzero, but as long as $v \varphi$ is the leading term, its effect is small. Under $\Theta$ conditions $(v=0), \mathrm{u} \approx \varphi^{2} / 2-\varphi^{\prime \prime} / 6$, and the nonlocal correction is relatively more important since $u$ is much smaller. Now eq 14 becomes

$$
\frac{\xi^{2}}{2} g^{\prime \prime}=g^{5}-g\left(1+\frac{\xi}{\sqrt{6}}\left(g^{2}\right)^{\prime \prime}\right)
$$

This equation was earlier derived by Van der Gucht et al.,32 with $\xi$ replaced by $\sqrt{2 / 3} \varphi_{\mathrm{b}}{ }^{-1}$. These authors showed that after multiplying by $\mathrm{g}^{\prime}$ and integration, $\mathrm{g}^{\prime}=\mathrm{dg} / \mathrm{dz}$ can be written as

$$
\frac{d g}{d(z / \xi)}=\sqrt{\frac{2 g^{6}-6 g^{2}+4}{3+\sqrt{24} g^{2} / \xi}}
$$

From eq 22 the function $z(g)$ may be found by numerical integration. An analytical solution is only possible when the nonlocal term $\mathrm{g}^{2} / \xi \sim \varphi_{\mathrm{b}} \mathrm{g}^{2}$ is neglected, corresponding to eq 21 in the simplified form $\left(\xi^{2} / 2\right) g^{\prime \prime}=g^{5}-g$. With the boundary condition $g(0)=0$, the solution is $\mathrm{s}^{32} \mathrm{~g}\left(2 \mathrm{~g}^{2}\right.$ $+1)^{-2}\left(3 g^{2}+6\right)^{-1 / 2}=t$, where $t=\tanh (\sqrt{8} z / \xi)$. Using the relation $\mathrm{t}^{2}=1-1 / \mathrm{c}^{2}$, with $\mathrm{c}=\cosh (\sqrt{8} z / \xi)$, we can

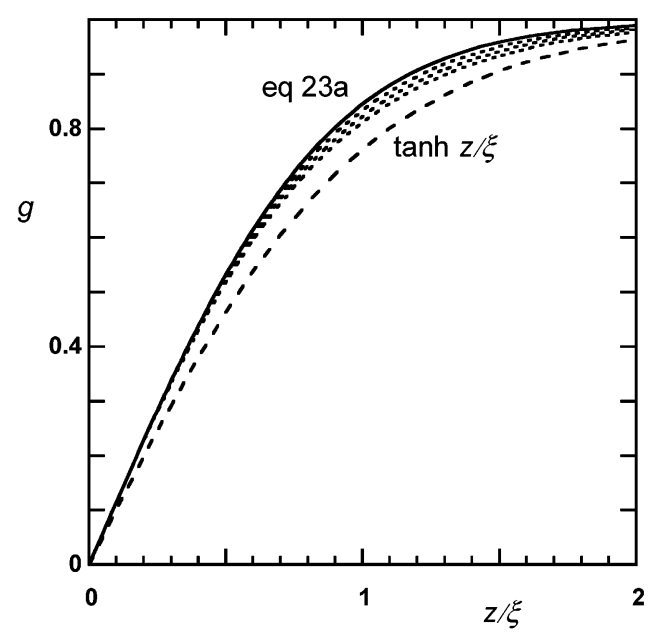

Figure 5. Ground-state function $g(z)$ in a $\Theta$ solvent. The solid curve (top) is the "Iocal" approximation eq 23a, and the dashed curve is the simplified form $\mathrm{g}=\tanh (\mathrm{z} / \xi)$. The dotted curves in between are the numerical solutions of eq 22 for, from top to bottom, $\varphi_{\mathrm{b}}=0.1,0.3$, and 0.5 .

rearrange it to $\left[(c+2) g^{2}-(c-1)\right]\left[(c-2) g^{2}-(c+1)\right]$ $=0$. Since $\mathrm{g}^{2}$ should be positive, the solution is $(c+2)$ $\mathrm{g}^{2}=\mathrm{c}-1$ or

$$
\begin{gathered}
g^{2}=\frac{\cosh (\sqrt{8} z / \xi)-1}{\cosh (\sqrt{8} z / \xi)+2} \\
\frac{\delta}{\xi}=\sqrt{\frac{3}{2}} \operatorname{atanh} \frac{1}{\sqrt{3}}=0.806
\end{gathered}
$$

Equation 23b was obtained by substituting $\rho=\mathrm{g}^{2}$ from eq 23a into eq 5.

Figure 5 (solid curve) gives the solution of eq 23 and also the numerical solution of eq 22 for $\varphi_{\mathrm{b}}=0.1,0.3$, and 0.5 (dotted). In this figure the full expression eq 16 was used for $\xi$. Also shown in Figure 5 is the simple form $\mathrm{g}=\tanh (\mathrm{z} / \xi)$ (dashed curve). This simple form, which gives $\delta=\xi$ according to eq 19 , is the correct solution in good solvents. The "local" solution eq 23 for a $\Theta$ solvent gives $\delta=0.81 \xi$ : the depletion zone is nearly $20 \%$ thinner than $\xi$ as given by eq 16 . The nonlocal term in eq 22 shifts the limiting curve of eq 23a (in which the nonlocal effect is neglected) outward, in the direction of the simple $\tanh (z / \xi)$, thus making $\delta$ slightly higher again. As an approximation, we will therefore use eq $18, \rho=\tanh ^{2}(z / \xi)$, and eq $19, \delta=\xi$, also in semidilute $\Theta$ solvents with, again, eq 16 for $\xi$. We see from Figure 5 that this is an overestimation of order $10 \%$ in $\delta$. In the Results section (Figure 8) where we make a comparison with the numerical SF model, we will indeed see such deviations.

We thus conclude that in semidilute solutions (where $\epsilon$ is zero) $\rho=g^{2}$ for flat plates is well approximated by eq 18, $\rho=\tanh ^{2}(z / \xi)$, where $\xi$ is only a function of concentration and solvency. This conclusion is valid for any solvency, provided $\xi$ is computed from eq 16 . In this way a smooth transition between the limiting cases $\xi^{-2}$ $=3 \mathrm{v} \varphi_{\mathrm{b}}$ (good solvent) and $\xi^{-2}=3 \varphi_{\mathrm{b}}{ }^{2} / 2$ ( $\Theta$ solvent) is obtained as a function of $\chi$. With this form of $\rho$, also eq $19, \delta=\xi$, is valid for any solvency and concentration in the semidilute regime, with the proviso that in a $\Theta$ solvent an overestimation of order $10 \%$ is expected. 


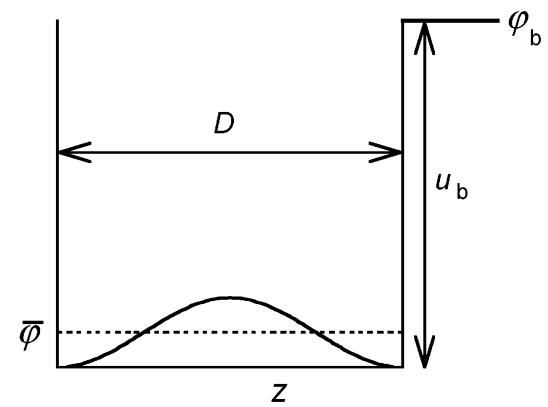

Figure 6. Concentration profile $\varphi(\mathrm{z})$ and its average value $\bar{\varphi}$ in a narrow slit of width $\mathrm{D}$, at an external concentration $\varphi_{\mathrm{b}}$. The slit may be consi dered as a region with a potential well of depth $\mathrm{u}_{\mathrm{b}}=-\ln \left(1-\varphi_{\mathrm{b}}\right)-2 \chi \varphi_{\mathrm{b}}$.

\section{General Equation for the Depletion Thickness}

We have now simple expressions for the depletion thickness in two limits: eq 8 gives $\delta=\delta(\mathrm{N})$ in (very) dilute solutions, and eq 19 in combination with eq 16 gives $\delta=\delta\left(\varphi_{\mathrm{b}}, \chi\right)$ in the semidilute case. In this section we bridge the gap between these two limits and propose a form that gives an analytical expression $\delta=\delta\left(\mathrm{N}, \varphi_{\mathrm{b}}, \chi\right)$ for intermediate cases as well. To that end, we extend the Casassa description given in section 3.2 by considering a nearly empty slit in contact with a solution of finite concentration $\varphi_{\mathrm{b}}$, using GSA. The slit may be seen as a region with a potential well of width $D$ and depth $u_{b}$ with respect to the bulk solution (see Figure 6). As long as the relative concentration $\bar{\rho}=\bar{\varphi} / \varphi_{\mathrm{b}}$ in the slit is small, we may approximate the depth of the well to be constant across the slit: we neglect $u /(z)$ with respect to $u_{b}$.

6.1. GSA for a Slit with $\mathbf{u}_{\mathbf{b}} \rightarrow \mathbf{0}$. To illustrate the principle, we first consider the GSA result for the case $\varphi_{\mathrm{b}} \rightarrow 0, \mathrm{u}_{\mathrm{b}} \rightarrow 0$, for which the exact solution is known (section 3.2). GSA (eq 14) gives $g^{\prime \prime}=6 \epsilon g$, where $\epsilon$ is negative. Rewriting this as $g^{\prime \prime}=-b^{2} g$, with $b^{2}=-6 \epsilon$, we see easily that the solution is $g=A$ sin bz. The constant $b$ (and therefore $\epsilon$ ) is determined by the boundary condition $g(0)=g(D)=0: b=\pi / D$ since the sine function should have half a period across the slit. Hence, $b$ and $\epsilon$ are only a function of the slit width. The prefactor $\mathrm{A}$ can be found from the requirement that the integral over $\rho_{\mathrm{e}}=\mathrm{N} \varphi_{\mathrm{d}} / \varphi_{\mathrm{b}}$ should give the same result as that over $\rho=\varphi / \varphi_{\mathrm{b}}$, since both integrals express the amount of polymer in the slit: each chain of $\mathrm{N}$ segments has exactly one end point with ranking number $\mathrm{S}=\mathrm{N}$. With eq 15, this translates into $\int g d z=\int g^{2} d z$, from which $A$ follows as $A=4 / \pi$. Hence, for $\varphi_{b} \rightarrow 0$ the GSA solution is

$$
\begin{gathered}
g=\frac{4}{\pi} \sin \frac{\pi z}{D} \\
\bar{\rho}=\frac{8}{\pi^{2}} e^{\epsilon N} \\
\epsilon=-\frac{\pi^{2}}{6 D^{2}}
\end{gathered}
$$

Using $N=6 R^{2}=(6 \pi / 4) \delta_{0}^{2}$ in eq $24 \mathrm{~b}$, we find exactly the narrow-pore limit eq 11 a for $\bar{\rho}$ as derived by Casassa. Equation 15b, in combination with eq 24b, reads $\rho=$ $\left(\pi^{2} / 8\right) \bar{\rho} \mathrm{g}^{2}$, which exactly reproduces eq $1 \mathrm{lb}$ for the profile. In this limit, the polymer concentration in the slit depends only on $\mathrm{D}$ and $\mathrm{N}$ (or $\delta_{0}$ ). Hence, for narrow empty slits with (very) low outside concentrations, GSA gives the same result as the exact Casassa treatment. Apparently, the first term in the full eq 10 corresponds to the ground state.

6.2. GSA for a Slit with $\mathbf{u}_{\mathbf{b}} \neq \mathbf{0}$. For nonzero $\varphi_{\mathrm{b}}$, small $\bar{\rho}$, and constant well depth $u_{b}$ across the slit (see Figure 6), this line of thought is easily generalized. The GSA equation is now $g^{\prime \prime}=6\left(\epsilon-u_{b}\right)$ g. Redefining $b^{2}=$ $-6 \epsilon+6 u_{b}$, we find again eqs $24 a$,b. However, eq $24 c$ is modified to $\epsilon=u_{b}-\pi^{2} / 6 D^{2}$ or, with eq 16

$$
\epsilon=\frac{1}{3 \xi^{2}}-\frac{\pi^{2}}{6 D^{2}}
$$

Using this simple operation of introducing a constant well depth $u_{\mathrm{b}}$, we see that $\epsilon$ is not only a function of $D$ but also of $\xi$ (hence of $\varphi_{\mathrm{b}}$ and $\chi$ ). Similarly, $\bar{\rho}$ is now not only a function of $\mathrm{D}$ and $\mathrm{N}$ but also of $\xi$.

For small $D, \epsilon$ in eq 25 is negative so that $\bar{\rho}$ in eq $24 b$ is small. With increasing $D, \epsilon$ and $\bar{\rho}$ increase. Equation 25 predicts $\epsilon$ to become zero at $D=(\pi / \sqrt{2}) \xi$. Clearly, at this point eq 25 breaks down as the slit is no longer empty, and the potential well in the slit has no longer a constant depth $u_{\mathrm{b}}$. In this limit of wide slits, we could in semidilute solutions use again $\epsilon=0$ and $\rho=\tanh ^{2}$ $(z / \xi)$ for both surfaces, leading to the semidilute equivalent of eq 12: $\bar{\rho}=1-2 \xi / \mathrm{D}$.

6.3. Derivation of $\delta(\mathbf{R}, \xi)$. Our central assumption in this paper is that eq 25 may be used to find an expression for the depletion thickness $\delta$ which has the limits $\delta=\delta_{0}$ for $\varphi_{\mathrm{b}} \rightarrow 0$ and $\delta=\xi$ for $\mathrm{N} \rightarrow \infty$ (semidilute), but which describes also intermediate situations. We assume that we may apply eq 25 for narrow pores up to $D \approx 2 \delta$, at which point we expect $\bar{\rho}$ in eq $24 \mathrm{~b}$ to be of order $\mathrm{e}^{-1}$. For comparison, when for $\varphi_{\mathrm{b}} \rightarrow 0$ (Figure 3) $\mathrm{D} \approx 2 \delta_{0}, \bar{\rho}$ is around 0.2 . When $\bar{\rho}$ is of order $-1, \epsilon \mathrm{N}=$ $6 \in \mathrm{R}^{2}$ in eq $24 \mathrm{~b}$ is of order -1 . Substituting $\mathrm{D} \approx 2 \delta$ and $6 \epsilon \approx-1 / \mathrm{R}^{2}=-(4 / \pi) / \delta_{0}^{2}$ into eq 25 , we find

$$
\frac{\pi^{2}}{8} \frac{1}{\delta^{2}} \approx \frac{2}{\pi} \frac{1}{\delta_{0}^{2}}+\frac{1}{\xi^{2}}
$$

Obviously, the numerical coefficients $\pi^{2} / 8$ and $2 / \pi$ are approximate. We can find more accurate values by considering the known limits. F or $\delta_{0} \rightarrow \infty$ we have the De Gennes limit $\delta=\xi$ (eq 19), so we drop the factor $\pi^{2} / 8$. Next, we apply $\delta=\delta_{0}$ for $\xi^{-2} \longrightarrow 0$ (eq 8) and find that we should also omit the factor $2 / \pi$. Hence, we end up with the extremely simple result

$$
\frac{1}{\delta^{2}}=\frac{1}{\delta_{0}^{2}}+\frac{1}{\xi^{2}}
$$

which describes the depletion thickness near a flat plate for any chain length, concentration, and solvency, using eq 8 for $\delta_{0}$ and eq 16 for $\xi$.

We note that Fleer et al. [ref 31, eq 4.4.18] derived a lattice expression for $\delta$ which may be cast in the form of eq 27. The derivation is based upon similar arguments as given above, using a discrete version of the differential equation $g^{\prime \prime}=6\left(\epsilon-u_{b}\right) g$ for a slit. This discrete form was solved along the lines of DiMarzio and Rubin, ${ }^{54}$ who gave the solution in the limit $u_{b} \rightarrow 0$. The result is eq 25 with $D$ replaced by $M+1$, where $M$ is the number of lattice layers in the slit. The difference is a shift over one bond length in the slit width, which leads to eq 27 with $\delta$ replaced by $\delta+0.5$ (and $\delta_{0}$ by $\delta_{0}$ 
$+0.5)$. In the appendix we show that this small shift is related to the boundary condition at the surface, where in the lattice model the degree of segment/surface repulsion has to be specified. By making the surface weakly repulsive, the boundary condition in the lattice model becomes the same as in the continuum description (i.e., $g(0)=0$ ) and the shift disappears: the lattice result is then equal to eq 27.

Equation 27 extends the De Gennes equation $\delta=\xi$ (where $\xi$ does not depend on chain length) to an $\mathrm{N}$-dependent depletion thickness $\delta$. There is a strong analogy with a generalized correlation length $\xi_{\mathrm{e}}$ as defined by Grossberg and Khoklov [ref 41, eq 24.7]: $\left(2 \xi_{\mathrm{e}}\right)^{-2}=3\left(1 / \mathrm{N}+\mathrm{d} \Pi / \mathrm{d} \varphi_{\mathrm{b}}\right)$, where $\mathrm{d} \Pi / \mathrm{d} \varphi_{\mathrm{b}}$ is the inverse of the osmotic compressibility (in kT units). We use the subscript e to indicate that this is the exponential decay length (blob radius). In a Flory-Huggins picture, $\mathrm{d} \Pi$ / $\mathrm{d} \varphi_{\mathrm{b}}=1 / \mathrm{N}+\varphi_{\mathrm{b}} /\left(1-\varphi_{\mathrm{b}}\right)-2 \chi_{\varphi_{\mathrm{b}}}{ }^{41} \mathrm{~F}$ or not too concentrated solutions, the concentration terms may be replaced by $\xi^{-2} / 3$, with $\xi$ defined as in eq 16 . Hence, $\left(2 \xi_{\mathrm{e}}\right)^{-2}$ $\approx 6 / \mathrm{N}+\xi^{-2} \approx \delta_{0}{ }^{-2}+\xi^{-2}$. We conclude that the depletion thickness $\delta$ can be considered as the $\mathrm{N}$-dependent correlation length (blob diameter) $2 \xi_{\mathrm{e}}$, which generalizes the statement below eq 19 that in semidilute solutions $\delta$ equals the $\mathrm{N}$-independent blob diameter $\xi$.

Using eq 27, we can now modify the Casassa result for the distribution coefficient (eq 10) simply by inserting $\delta=\delta\left(R, \chi_{1} \varphi_{\mathrm{b}}\right)$ instead of $\delta_{0}=\delta_{0}(\mathrm{R})$ :

$$
\bar{\rho}=\frac{8}{\pi^{2}} \sum_{n=1,3 \ldots} \frac{1}{n^{2}} e^{-n^{2} \pi^{3}(\delta / 2 D)^{2}}
$$

The application of this equation to the concentration dependence in the separation of polymers in size exclusion chromatography will be discussed in a next paper.

\section{Extension to Spheres}

7.1. Dilute Limit. In section 3.1 (eq 6) we gave the exact zero-field expressions for the concentration profile next to a flat wall. The generalization to spheres of radius a is slightly more complicated. ${ }^{12,13}$ The distance $z$ from the sphere surface equals $r-a$. Using the subscript $\mathrm{s}$ for the profile around a sphere, we may write $\rho_{\text {se }}(z)$ (ends) and $\rho_{s}(z)$ (overall) as ${ }^{12,13}$

$$
\begin{gathered}
\rho_{\mathrm{se}}=\frac{\mathrm{z} / \mathrm{a}+\rho_{\mathrm{e}}(\xi)}{\mathrm{z} / \mathrm{a}+1} \\
\rho_{\mathrm{s}}=\frac{(\mathrm{z} / \mathrm{a})^{2}+2(\mathrm{z} / \mathrm{a}) \psi(\zeta)+\rho(\zeta)}{(\mathrm{z} / \mathrm{a}+1)^{2}}
\end{gathered}
$$

where $\rho_{\mathrm{e}}(\xi)$ (ends, eq 6a) and $\rho(\xi)$ (overall, eq 6b) are the profiles at a flat wall, with $\zeta=z / 2 \mathrm{R}$, and $\psi$ in eq $29 \mathrm{~b}$ is given by eq 7 . Clearly, for $\mathrm{a} \rightarrow \infty$ eq 29 reduces to eq 6.

The exact eq $29 \mathrm{~b}$ is rather cumbersome because of the complicated form of the function $\psi(\zeta)$, which also enters $\rho(\xi)=2 \psi(\xi)-\psi(2 \xi)$. In section 3.1 and $\mathrm{Figure} 1$ we saw that $\psi(\xi) \approx \tanh (\xi \sqrt{\pi})$ and $\rho(\zeta) \approx \tanh ^{2}(\xi \sqrt{\pi})$. With these approxi mations, the numerator of eq $29 \mathrm{~b}$ reduces to the square of $z / a+\tanh (\xi \sqrt{\pi})=z / a+\tanh \left(z / \delta_{0}\right)$. Hence, the rather complicated eq $29 \mathrm{~b}$ may be rewritten in the approximate but very handy form

$$
\rho_{\mathrm{s}}=\left(\frac{\mathrm{z} / \mathrm{a}+\tanh \left(\mathrm{z} / \delta_{0}\right)}{\mathrm{z} / \mathrm{a}+1}\right)^{2}
$$

In the flat-plate limit this reduces to eq 9: $\rho=\tanh ^{2}$ $\left(z / \delta_{0}\right)$, where $\delta_{0}$ is only a function of chain length (eq 8).

7.2. Semidilute Limit. We assume that the transformation from eq 9 (flat) to eq 30 (spheres), which is valid in dilute solutions, also holds in semidilute solutions. The semidilute flat-plate equivalent of eq 9 is eq 18: $\rho=\tanh ^{2}(z / \xi)$, where $\xi$ does not depend on chain length but is a function of concentration and solvency. Then the semidilute equivalent of eq 30 is

$$
\rho_{\mathrm{s}}=\left(\frac{\mathrm{z} / \mathrm{a}+\tanh (\mathrm{z} / \xi)}{\mathrm{z} / \mathrm{a}+1}\right)^{2}
$$

The same form was proposed by Tuinier and Lekkerkerker. ${ }^{43}$ These authors tested this equation against the numerical solution of the spherical analogue of eq 17 for good solvents (with g" replaced by $\nabla^{2}$ g in spherical coordinates and for $\xi^{-2}$ the limiting form $\left.3 \mathrm{v} \varphi_{\mathrm{b}}\right)$. Excellent agreement was found for $\xi / a$ of order 0.1 (the socalled colloid limit) and for $\xi / a$ of order 50 (protein limit). In the intermediate range the agreement is satisfactory, with an underestimation of $\rho_{\mathrm{s}}$ according to eq 31 of a few percent. Tuinier and Lekkerkerker suggested a correction on eq 31 that does indeed work better but is more invol ved and cannot be written in the simple form of eq 30 (dilute) or eq 31 (semidilute). For convenience, we stick to the simple transparent form of eq 31, accepting some minor deviations.

7.3. Generalization. It is now straightforward to generalize eq 30 (with $z / \delta_{0}$, valid for $\varphi_{\mathrm{b}} \rightarrow 0$ ) and eq 31 (with $z / \xi$, applicable in semidilute solutions) to a wide range of concentrations:

$$
\rho_{\mathrm{s}}=\left(\frac{\mathrm{z} / \mathrm{a}+\tanh (\mathrm{z} / \delta)}{\mathrm{z} / \mathrm{a}+1}\right)^{2}
$$

where $\delta=\delta\left(\mathrm{R}, \chi_{,} \varphi_{\mathrm{b}}\right)$ is given by eq 27. In the Results section, we will check this simple result against two numerical models: that of Schaink and Smit ${ }^{53}$ (a GSAtype continuum theory based upon the square-gradient model, which neglects nonlocal energy effects) and the discrete numerical SF model. ${ }^{29-32}$

7.4. Depletion Thickness around a Sphere. In eq 5 the depletion thickness at a flat plate was defined as the zeroth moment of the concentration profile or, equivalently, $\delta$ was related to the negative adsorption $-\theta^{e} \times / \varphi_{\mathrm{b}}$. In other geometries the negative adsorption also yields the depletion thickness. The depletion thickness $\delta_{\mathrm{s}}$ around a sphere of radius a follows from

$$
-\frac{\Gamma}{\varphi_{\mathrm{b}}}=\frac{4 \pi}{3}\left(\left(\mathrm{a}+\delta_{\mathrm{s}}\right)^{3}-\mathrm{a}^{3}\right)=4 \pi \int_{\mathrm{a}}^{\infty} \mathrm{r}^{2}\left(1-\rho_{\mathrm{s}}\right) \mathrm{dr}
$$

where $r$ is the radial distance from the center of the sphere and $\Gamma$ the total negative adsorption around the sphere; we use again the subscript $\mathrm{s}$ in $\rho_{\mathrm{s}}$ to indicate the spherical symmetry. The parameter $z$ as used for planar geometry is related to $r$ through $z=r-a$. When exact expressions for $\rho_{\mathrm{s}}(r)$ (overall) and $\rho_{\mathrm{se}}(r)$ (ends) are available which satisfy eqs 3 and 4 (such as given in eq 29 for the dilute limit), the quantity $\rho_{s}$ in eq 33 could be replaced by $\rho_{\text {se }}$ with the same result.

Equation 33 gives the relation between the depletion thickness $\delta_{\mathrm{s}}$ around a sphere and the concentration profile. When $\rho_{s}(z)=\rho_{s}(r-a)$ is described through eq 32 , in which the only parameter is the depletion thickness $\delta$ at a flat wall, we find from eq 33 a simple relation between $\delta_{\mathrm{s}}$ and $\delta$ : 


$$
\left(1+\frac{\delta_{s}}{\mathrm{a}}\right)^{3}=1+3 \frac{\delta}{\mathrm{a}}+\frac{\pi^{2}}{4}\left(\frac{\delta}{\mathrm{a}}\right)^{2}
$$

from which, using eq $27, \delta_{\mathrm{s}}$ may be computed for any particle radius $\mathrm{a}$, any concentration $\varphi_{\mathrm{b}}$, any solvency $\chi_{\text {, }}$ and any chain length $\mathrm{N}=6 \mathrm{R}^{2}$. For $\mathrm{a} \rightarrow \infty, \delta_{\mathrm{s}}$ is equal to $\delta$, but for small particle sizes $\delta_{s} / a$ scales as $(\delta / a)^{2 / 3}$, or $\delta_{s} / \delta \sim(\delta / a)^{-1 / 3}$. Figure 7 gives a double-logarithmic plot of $\delta_{\mathrm{s}} / \delta$ as a function of a/ $\delta$. The limiting behaviors $\delta_{\mathrm{s}} / \delta$ $\sim(\mathrm{a} / \delta)^{1 / 3}$ in the protein limit and $\delta_{\mathrm{s}}=\delta$ in the colloid limit are clearly seen. The dotted line in Figure 7 is the asymptote $\delta_{s} / \delta=(\pi / 2)^{2 / 3}(\mathrm{a} / \delta)^{1 / 3}$. We note that $\delta=\delta\left(\delta_{0}, \xi\right)$ is independent of the particle radius, whereas $\delta_{\mathrm{s}}=$ $\delta_{s}\left(\delta_{0}, \xi, a\right)$ is not. We intend to discuss the implications for colloid stability in a different paper.

We note that Aarts et al. ${ }^{25}$ applied eq 33 with the exact expression $29 \mathrm{~b}$ for $\rho_{\mathrm{s}}$ in the dilute limit. Their result is nearly identical to eq 34 . The only difference is that the factor $\pi^{2} / 4$ in the third term is replaced by $3 \pi / 4$, which is smaller by a factor $\pi / 3$. When exact agreement with the dilute limit is desired, we could introduce this factor $\pi / 3$ into eq 34 and thus use the expression of Aarts et al. with $\delta_{0}$ replaced by $\delta$.

According to eq 33 , the excess amount $\theta_{\mathrm{s}}^{\mathrm{ex}}=\Gamma / 4 \pi \mathrm{a}^{2}$ per unit area around a sphere is given by $-\left(\varphi_{\mathrm{b}} \mathrm{a} / 3\right)[(1+$ $\left.\left.\delta_{s} / a\right)^{3}-1\right]$. Combining this with eq 34 , we find

$$
-\frac{\theta_{\mathrm{s}}^{\mathrm{ex}}}{\varphi_{\mathrm{b}}}=\delta\left(1+\frac{\pi^{2}}{12} \frac{\delta}{\mathrm{a}}\right)
$$

For $\mathrm{a} \rightarrow \infty$, this reduces to $-\theta^{\mathrm{ex}} / \varphi_{\mathrm{b}}=\delta$, which is eq 5 . We see from eq 35 that the excess amount per unit area around a sphere can be expressed only in the particle radius a and the depletion thickness $\delta$ at a flat plate, which is given by eq 27 .

Equation 35, as all results in this paper, is a meanfield result. A similar general expression for the exdudedvolume limit is not known. However, Eisenriegler et al. ${ }^{13,21,55}$ derived such a result for the protein limit: $-\Gamma$ $=\mathrm{F}\left(\mathrm{d} \Pi / \mathrm{d} \varphi_{\mathrm{b}}\right)^{-1}$, where $\mathrm{d} \Pi / \mathrm{d} \varphi_{\mathrm{b}}$ is the inverse of the osmotic compressibility, equal to $1 / \mathrm{N}$ in dilute solutions, and $F$ is the free energy of immersing a (small) sphere into a polymer solution (in kT units). For mean-field chains $\left(R \approx N^{0.5}\right), F$ is given by $F=4 \pi\left(\varphi_{b} / N\right) a R^{2}$, where $\varphi_{\mathrm{b}} / \mathrm{N}$ is the number concentration of polymer chains. ${ }^{21,55}$ Hence, the exact E isenriegler result for dilute solutions in the protein limit is $-\Gamma / \varphi_{\mathrm{b}}=4 \pi \mathrm{aR} \mathrm{R}^{2}$. Equation 35 for small a reads $-\Gamma / \varphi_{\mathrm{b}}=\left(\pi^{3} / 3\right) \mathrm{a} \delta^{2}=\left(4 \pi^{2} / 3\right) \mathrm{aR} \mathrm{R}^{2}$, which is nearly the same result with, again, a factor $\pi / 3$ difference. It is therefore possible to replace $\pi^{2} / 12$ in eq 35 by $\pi / 4$ in order to match the exact solution in the dilute limit.

In a similar vein, the profile around a small sphere as given by eq 32 may be compared with an exact expansion in the excluded-volume limit for distances $r$ much larger than the sphere radius a. ${ }^{55}$ It may be shown ${ }^{56}$ that eq 32 is consistent with such an expansion for arbitrary chain overlap, all the way from dilute to semidilute solutions.

E quation 33 may be written as $\Gamma=\int \gamma d r$, where $\gamma(r)$ $\mathrm{dr}$ is the deficit of polymer in a spherical shell of radius $r=a+z$ and thickness dr. According to eq 33, $\gamma$ is given by $\gamma=4 \pi \mathrm{r}^{2}\left(\varphi-\varphi_{\mathrm{b}}\right)=4 \pi \mathrm{r}^{2}\left(\rho_{\mathrm{s}}-1\right) \varphi_{\mathrm{b}}$. When we divide $\gamma$ by the area $4 \pi \mathrm{a}^{2}$ of the sphere, we may define the quantity $\rho_{\mathrm{s}}^{\mathrm{ex}}(\mathrm{z})=\gamma / 4 \pi \mathrm{a}^{2} \varphi_{\mathrm{b}}=\left(\mathrm{r}^{2} / \mathrm{a}^{2}\right)\left(\rho_{\mathrm{s}}-1\right)$, where $\rho_{\mathrm{s}}^{\mathrm{ex}}$ is the contribution of the shell between $r$ and $r+d r$ to

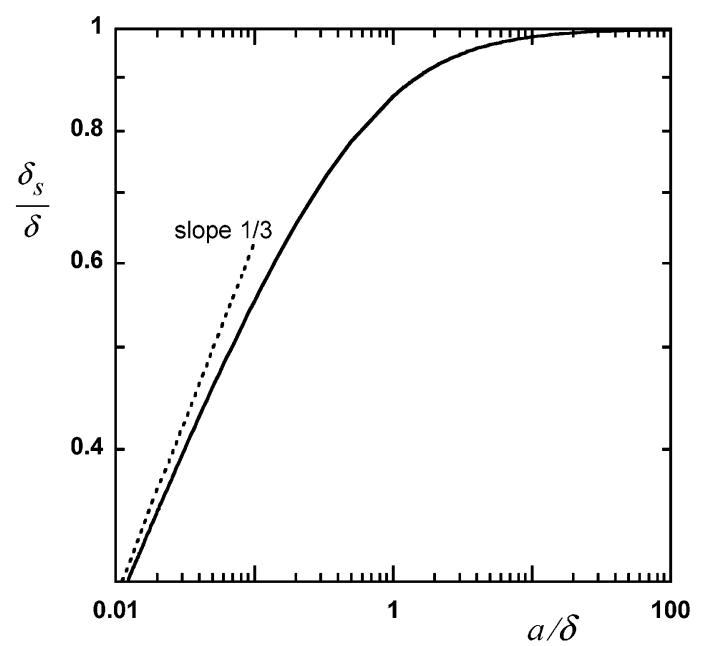

Figure 7. Ratio $\delta_{s} / \delta$ between the depletion thickness $\delta_{\mathrm{s}}$ around a sphere of radius a and the depletion thickness $\delta$ at a flat plate, as a function of the ratio $a / \delta$. The dashed line is the asymptote $\delta_{s} / \delta=(\pi / 2)^{2 / 3}(\mathrm{a} / \delta)^{1 / 3}$, corresponding to the protein limit.

the total negative adsorption $\theta_{\mathrm{s}}^{\mathrm{ex}} / \varphi_{\mathrm{b}}$ (per unit area), which is given by eq 35 . Using eq 32 for $\rho_{\mathrm{s}}$, we find

$$
\rho_{\mathrm{s}}^{\mathrm{ex}}=\frac{2 \mathrm{z}}{\mathrm{a}}\left(\tanh \frac{\mathrm{z}}{\delta}-1\right)+\tanh ^{2} \frac{\mathrm{z}}{\delta}-1
$$

Obviously, integration of eq 36 gives eq 35 . In the limit $\mathrm{a} \rightarrow \infty,\left|\rho_{\mathrm{s}}^{\mathrm{ex}}\right|$ equals $1-\tanh ^{2}(\mathrm{z} / \delta)$, which is a monotonically decreasing function of $z$, whereas for small a the first term dominates: $\left|\rho_{\mathrm{s}}^{\mathrm{ex}}\right|=(2 z / a)[1-\tanh (z / \delta)]$, giving a curve with a maximum as a function of $z$. In section 8.4 (Figure 12), we give a plot of $\left|\rho_{\mathrm{s}}^{\mathrm{ex}}\right|$.

The excess amount is closely related to the surface free energy, to the phase behavior of colloids in solutions of nonadsorbing polymer, and to the partition coefficient in size-exclusion chromatography. We shall elaborate on these aspects elsewhere.

\section{Results}

8.1. Mapping of Continuum and Lattice Models. The central equation in this paper is eq 27, $\delta^{-2}=\delta_{0}{ }^{-2}$ $+\xi^{-2}$. We will check this approximate GSA result against the numerical SF model in section 8.2. In this comparison, the boundary condition $\mathrm{g}(0)$ used to derive eq 27 must be translated into a lattice boundary condition, which means that the value of the segment/ surface repulsi on has to be specified. In a lattice model, this repulsion is expressed through the parameter $\Delta \chi$ $=\chi_{\mathrm{PS}}-\chi_{\text {, where }} \chi_{\mathrm{PS}}$ is the Flory-Huggins parameter for the interaction between polymer segments and units of the solid. In the depletion range $(\Delta \chi \geq 0)$ there is only little effect of an additional repulsion due to the wall, but nevertheless the depletion thickness for $\Delta \chi=\infty$ (strongly repulsive surface) is one bond length higher than for $\Delta \chi=0$ (neutral surface). The appendix gives some detail. In this appendix it is shown that the boundary condition $g(0)=0$ in the continuum model corresponds to a weakly repulsive surface in the lattice model, with $\Delta \chi=1$. With this choice of $\Delta x$, the depletion thickness is the same in both models. Ther efore, all the SF results presented in this paper were computed with $\Delta \chi=1$.

8.2. Check of Our General Equation. According to eq 8 , in the dilute limit $\delta=\delta_{0}$ depends only on chain length and is independent of concentration and solvency. 


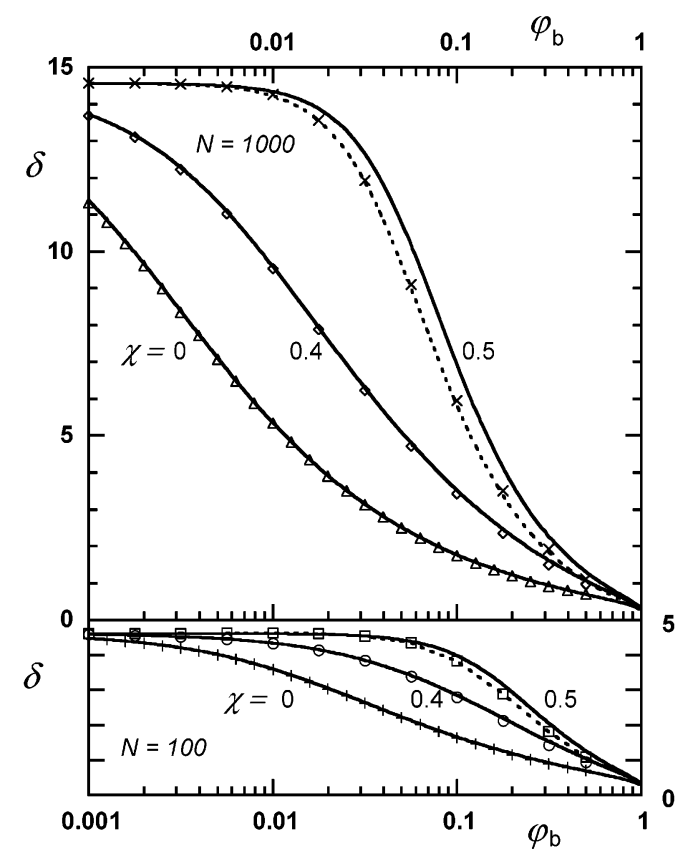

Figure 8. Depletion thickness at a flat wall according to eq 27 (solid curves) as compared to the SF results (symbols), for two chain lengths (upper part $N=1000$, lower part $N=100$ ) and three sol vencies $\chi=0,0.4$, and 0.5 . The dotted curves for a $\Theta$ solvent $(\chi=0.5)$ correspond to eq 27 with $\xi$ replaced by $0.81 \xi$, which is the "local" solution eq $23 \mathrm{~b}$.

From eq 27 it follows that at finite concentrations $\delta$ becomes also a function of $\varphi_{\mathrm{b}}$ and $\chi_{\mathrm{r}}$, with the $\mathrm{N}$ dependence disappearing in the semidilute limit. Figure 8 gives $\delta$ as a function of $\log \varphi_{\mathrm{b}}$ for two chain lengths, $\mathrm{N}=100$ and $\mathrm{N}=1000$, and for three solvencies, $\chi=0$, 0.4 , and 0.5 . The curves were computed from eq 27 , and the symbols in Figure 8 are SF results. In order not to overcrowd the figure, we plotted the data for $\mathrm{N}=100$ at the bottom part, using the same scale as for $\mathrm{N}=1000$ in the main figure.

F or $\chi=0$ and $\chi=0.4$ the agreement is nearly perfect over the whole concentration range, which is a strong indication that eq 27 is sound. For $\chi=0.5$ the agreement is excellent at low concentrations where $\delta \approx \delta_{0}$, but slightly less when the $\xi^{-2}$ contribution becomes important. In this region eq 27 overestimates $\delta$ by about 10\%, as anticipated in section 5.3. The reason is the simplification of using the tanh ${ }^{2}$ also in a $\Theta$ solvent, which predicts a slightly wider depletion zone than the GSA solution (eqs 22 and 23) for a semidilute $\Theta$ solvent (see Figure 5). This is corroborated when, for a $\Theta$ solvent, we replace in eq $27 \xi$ by $0.81 \xi$ (see eq $23 \mathrm{~b}$ ), which corresponds to the solution of the "local" differential equation $\left(\xi^{2} / 2\right) g^{\prime \prime}=g^{5}-g$. This solution is indicated in Figure 8 as the dotted curve. The numerical SF data are in between the two limiting curves due to the nonlocal effect, but are rather close to the "local" solution. However, even the "universal" form based upon $\delta \approx \xi$ in semidilute $\Theta$ solvents predicts the trends quite well.

In agreement with expectation, $\delta$ is proportional to $\sqrt{\mathrm{N}}$ in dilute solutions where the $\xi^{-2}$ term is small and independent of $\mathrm{N}$ in semidilute solutions where $\xi^{-2}$ dominates. In the transition region $\delta$ decays relatively steeply. The location of this transition region may be characterized by the midpoint $\delta=\delta_{0} / 2$, where $\delta_{0}^{2}=3 \xi_{\mathrm{m}}^{2}$ or $\xi_{\mathrm{m}}=(2 / 9 \pi)^{1 / 2} \sqrt{\mathrm{N}}$, with $\xi_{\mathrm{m}}$ the value of $\xi$ at the

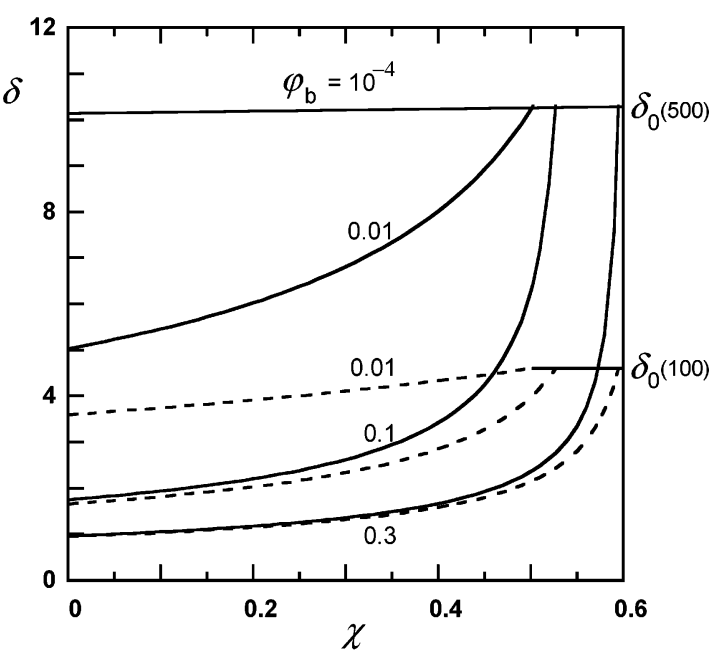

Figure 9. Depletion thickness $\delta$ at a flat plate (eq 27) as a function of the solvent quality $\chi$, for $\mathrm{N}=500$ (solid curves) and $\mathrm{N}=100$ (dashed), and at three concentrations, as indicated. The curves reach the limit $\delta_{0}$ (indicated to the right) at $\chi=0.5,0.503,0.527$, and 0.594 for the four concentrations used in the figure.

midpoint. For a given $\mathrm{N}$, the midpoint of the transition can thus be read from a horizontal cross section of Figure 4. For $\mathrm{N}=1000$, its value is $\xi_{\mathrm{m}}=8.41$, corresponding to midpoint concentrations $\varphi_{\mathrm{m}}=0.0047$ for $\chi=0,0.022$ for $\chi=0.4$, and 0.094 for $\chi=0.5$. For $\mathrm{N}$ $=100, \xi_{\mathrm{m}}=2.66$, and these midpoints are at $\varphi_{\mathrm{m}}=0.046$, 0.16 , and 0.28 , respectively. These numbers may also be interpreted as follows. Since $\xi_{\mathrm{m}} \sim \sqrt{\mathrm{N}}$, we have $\xi_{\mathrm{m}}{ }^{-2}$ $\sim \mathrm{N}^{-1}$. Hence, for a good solvent $\mathrm{v} \varphi_{\mathrm{m}} \sim \mathrm{N}^{-1}$ or $\varphi_{\mathrm{m}} \sim$ $(\mathrm{vN})^{-1}$. Indeed, in Figure 8 there is a factor of 10 in $\varphi_{\mathrm{m}}$ between the two chain lengths for $\chi=0$. For $\chi=0.4$ this ratio is somewhat higher because $\varphi_{\mathrm{m}}$ for $\mathrm{N}=100$ is outside the range where the asymptote $\xi^{-2}=3 \mathrm{v} \varphi_{\mathrm{b}}$ holds. The ratio in $\varphi_{\mathrm{m}}$ between the two solvencies is indeed 5 for $\mathrm{N}=1000$, as expected, but slightly more for $\mathrm{N}=100$ because the asymptote is inaccurate. For a $\Theta$ solvent $\varphi_{\mathrm{m}}{ }^{2} \approx \mathrm{N}^{-1}$ or $\varphi_{\mathrm{m}} \approx \mathrm{N}^{-1 / 2}$, so we observe only a factor $\sqrt{10}$ between the two chain lengths.

The slope of the curves in Figure 8 is easily derived from eq 27. At fixed $\delta_{0}, \mathrm{~d} \delta^{-2} / \mathrm{d} \xi^{-2}=1$ or $\mathrm{d} \delta / \mathrm{d} \xi=(\delta / \xi)^{3}$. Therefore $\mathrm{d} \delta / \mathrm{d} \ln \varphi_{\mathrm{b}}=(\delta / \xi)^{3} \mathrm{~d} \xi / \mathrm{d} \ln \varphi_{\mathrm{b}}$, where $\mathrm{d} \xi / \mathrm{d} \ln$ $\varphi_{\mathrm{b}}$ equals $-\xi / 2$ in a good solvent and $-\xi$ in a $\Theta$ solvent. The slope at the midpoint is found by inserting $\delta=\delta_{0} / 2$ and $\xi=\delta_{0} / \sqrt{3}$, which gives $\mathrm{d} \delta / \mathrm{d} \log \varphi_{\mathrm{b}}=-2.3(3 / 16) \delta_{0}$ in a good solvent and $-2.3(3 / 8) \delta_{0}$ for $\Theta$ conditions. Hence, this slope is proportional to $\sqrt{\mathrm{N}}$, and it is the same for $\chi=0$ and $\chi=0.4$. For $\chi=0.5$, it is twice that in a good solvent: the transition is steeper.

8.3. Effect of Solvency and Chain Length. In Figure 8 it is clearly visible that, for finite solution concentrations, the depletion thickness $\delta$ decreases with increasing solvency (decreasing $\chi$ ). The reason is the increasing osmotic pressure of the solution, which pushes the chains toward the surface; this tendency is highest at $\chi=0$, leading to a narrow depletion zone and a small $\delta$. Figure 9 gives a plot of $\delta$ as a function of $\chi$ for four concentrations and two chain lengths: $N=100$ (dashed curves) and $\mathrm{N}=500$ (solid curves). We have plotted each curve for the range in $\chi$ over which our model is expected to hold, which is for $u_{b} \geq 0, \xi^{-2} \geq 0$. The upper limit $\chi_{\mathrm{m}}$ is found from setting $\mathrm{u}_{\mathrm{b}}=0$ in eq 2b: $\chi_{\mathrm{m}}=-\left(2 \varphi_{\mathrm{b}}\right)^{-1} \ln \left(1-\varphi_{\mathrm{b}}\right) \approx 0.5\left(1+\varphi_{\mathrm{b}} / 2+\ldots\right)$. This value of $\chi_{\mathrm{m}}$ is very close to the phase separation condi- 


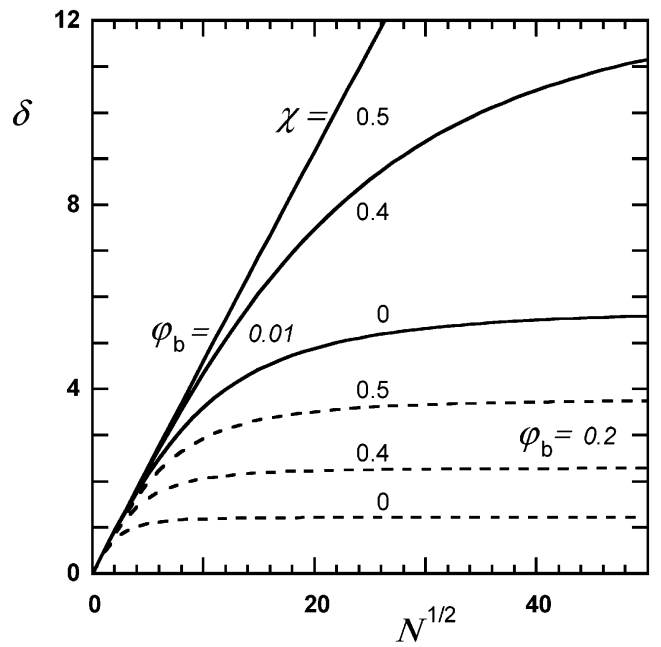

Figure 10. Depletion thickness $\delta$ at a flat plate (eq 27) as a function of $\sqrt{\mathrm{N}}$ for $\varphi_{\mathrm{b}}=0.01$ (solid curves) and $\varphi_{\mathrm{b}}=0.2$ (dashed) and for three solvencies, as indicated.

tions for infinite chain length. At this point our model predicts $\delta=\delta_{0}$, which is the E isenriegler result (eq 8). Note, however, that eq 8 was derived for zero $u_{b}$ and $\mathrm{u}(\mathrm{z})$. The value of $\delta_{0}$ (4.61 for $\mathrm{N}=100$ and 10.30 for $\mathrm{N}$ $=500$ ) is indicated in Figure 9 . F or the four concentrations used in Figure 9, $\chi_{\mathrm{m}}$ equals $0.5\left(10^{-4}\right), 0.503$ (0.01), $0.527(0.1)$, and 0.594 (0.3). Solutions of relatively short chains are stable for $\chi$ values slightly beyond $\chi_{\mathrm{m}}$, but in that region eq 16 breaks down. We may expect that in that narrow range the Eisenriegler result $\delta \approx \delta_{0}$ is a reasonable approximation.

At the lowest concentration $\left(\varphi_{\mathrm{b}}=10^{-4}\right)$ in Figure 9, the field is so low that $\xi^{-2}$ in eq 27 does not contribute so we have $\delta \approx \delta_{0}$ for any solvency. In our mean-field picture, chain swelling in good solvents is neglected so we do not capture the well-known effect that $R$ and, hence, $\delta$ in (extremely) dilute solutions increase with increasing solvency. We have shown the horizontal line $\delta=\delta_{0}$ for $\varphi_{\mathrm{b}}=10^{-4}$ at $\mathrm{N}=100$ only for the region $\chi>$ 0.5 . F or higher concentrations we see the expected effect of the increasing osmotic pressure, which makes $\delta$ much smaller in good solvents. For $\varphi_{\mathrm{b}}=0.01$ and $\mathrm{N}=100, \delta$ $\approx 0.5 \delta_{0}$ at $\chi=0$, but for $\mathrm{N}=100$ the relative effect is much smaller. At the highest concentration $\left(\varphi_{\mathrm{b}}=0.3\right)$ in Figure 9 we are in the concentrated regime where $\delta$ $\approx \xi$, and the lower curves in this figure, which are essentially independent of $\mathrm{N}$ for $\chi<0.5$, reflect the $\chi$ dependence of $\xi$ (see Figure 4). However, for $0.5<\chi<$ $\chi_{\mathrm{m}}, \delta$ approaches $\delta_{0}$, which depends on chain length. For $\varphi_{\mathrm{b}}=0.1$ the curves are intermediate between those for 0.01 and 0.3 , with semidilute ( $\mathrm{N}$-independent) behavior at low $\chi$ and the effect of the chain length visible at lower $\chi$ than for the higher concentration $\varphi_{\mathrm{b}}=0.3$.

Figure 10 shows the chain-length dependence of $\delta$ for $\varphi_{\mathrm{b}}=0.01$ (solid curves) and $\varphi_{\mathrm{b}}=0.2$ (dashed) and $\chi=$ $0.5,0.4$, and 0 . For $\varphi_{\mathrm{b}}=0.01, \delta \approx \delta_{0}$ for $\chi=0.5$ and $\mathrm{N}$ $<1000$ so that $\delta$ is proportional to $\sqrt{\mathrm{N}}$ (at least in the range shown in Figure 10; for $\mathrm{N} \rightarrow \infty$ a plateau at $\xi=$ $\sqrt{2 / 3} \varphi_{\mathrm{b}}{ }^{-1}=82$ is reached). The curves for $\chi=0.4$ and $\chi$ $=0$ start off in the same fashion for low $\mathrm{N}$ but then flatten because the $\xi^{-2}$ contribution to $\delta^{-2}$ is becoming more important. This contribution dominates at high $\mathrm{N}$, so that a plateau is reached at $\delta=\xi$. For $\varphi_{\mathrm{b}}=0.2$, this plateau extends over nearly the entire chain-length range, with the highest $\delta=\xi$ at the highest $\chi$.

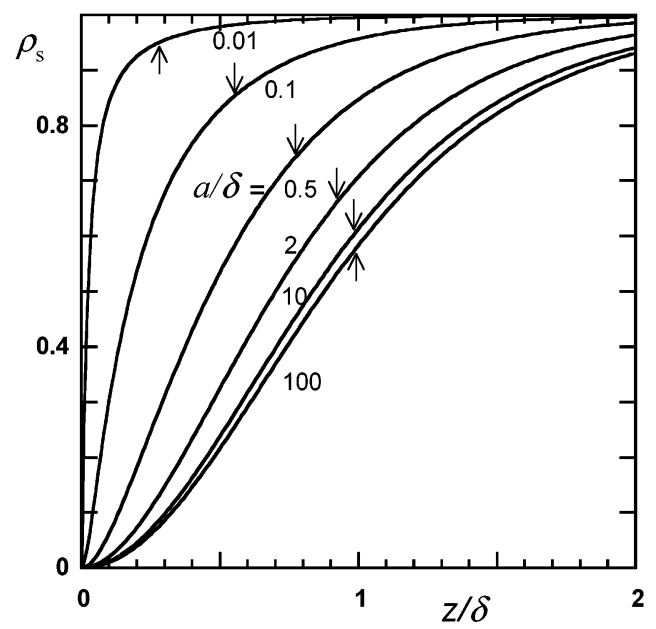

Figure 11. Concentration profiles around a sphere of radius a (eq 32) as a function of the scaled distance $z / \delta$ for various ratios $a / \delta$, ranging from the protein limit $(a / \delta=0.01)$ to the colloid limit $(\mathrm{a} / \delta=100)$. The value of the ratio $\delta_{s} / \delta$ is indicated by the arrows.

All the curves in Figure 10 have the following characteristic shape. Suppose we plot $\delta$ against $\delta_{0}$ (which is proportional to $\sqrt{\mathrm{N}}$ ). Such a plot has two asymptotes: $\delta=\delta_{0}$ (a $45^{\circ}$ line starting from the origin) and $\delta=\xi$ (a horizontal final level). The two asymptotes intersect at the point $\delta=\delta_{0}=\xi$. Each curve in Figure 10 follows initially the $\delta_{0}$ asymptote and ends up on the $\xi$ asymptote, with a round-off around the intersection point. For $\delta_{0}=\xi$ (the abscissa value of the intersection point) $\delta=\xi / \sqrt{2}$, which is $71 \%$ of the ordinate value of the intersection point.

8.4. Normalized Profiles around a Sphere. Figures 8-10 illustrate the dependence $\delta\left(\mathrm{N}, \varphi_{\mathrm{b}}, \chi\right)$ for the depletion thickness $\delta$ at a flat plate. In all cases the concentration profiles are given by $\rho=\tanh ^{2}(z / \delta)$. In Figure 11 we give the profiles $\rho_{\mathrm{s}}(\mathrm{z})$ around a sphere for various particle radii $a$, according to eq 32 . Both the distance from the surface and the particle radius a are scaled on $\delta$. F or $a / \delta=100$ we have essentially the flat profile $\rho=\tanh ^{2}(z / \delta)$; this is the so-called colloid limit. With decreasing $a / \delta$ the profiles move inward; the depletion layer becomes thinner. This is related to the decreasing value of $\delta_{s} / \delta$ (see Figure 7 ) as a/ $\delta$ becomes smaller. This ratio $\delta_{s} / \delta$ is unity for a/ $\delta \rightarrow \infty$ (flat wall) and becomes proportional to $a / \delta^{1 / 3}$ for (very) small $a / \delta$ (protein limit). For the six values of $a / \delta$ used in Figure $11, \delta_{s} / \delta$ equals $0.998,0.983,0.921,0.782,0.552$, and 0.282 . These values are indicated along the curves of Figure 11 by an arrow.

Figure 11 gives the local concentration at some point at a distance $z$ from the sphere. The total negative adsorption in a spherical shell of radius $r=a+z$ is related to excess quantity $\rho_{s}^{\mathrm{ex}}$, defined in eq 36 . Figure 12 shows $\left|\rho_{\mathrm{s}}^{\mathrm{ex}}\right|$ (on a logarithmic scale) as a function of $z / \delta$ for (from top to bottom) $a / \delta=0.01,0.05,0.1,0.2$, $0.5,2$, and 10 . The corresponding values of $\delta_{\mathrm{s}} / \delta(0.282$, $0.485,0.552,0.653,0.782,0.921$, and 0.983 , respectively) are again indicated as the arrows. The dashed curve in Figure 12 is the "flat" limit $\left|\rho_{\mathrm{s}}^{\mathrm{ex}}\right|=1-\rho=1-$ tanh$^{2}$ $(\mathrm{z} / \delta)$, corresponding to $\mathrm{a} / \delta=\infty$. The curves for small $\mathrm{a} / \delta$ pass through a maximum, as anticipated in the discussion below eq 36 (section 7.4). It is interesting to note that, although $\delta_{\mathrm{s}}$ decreases as the particles become smaller, the excess $\left|\theta_{\mathrm{s}}^{\mathrm{ex}} / \varphi_{\mathrm{b}}\right|$ per unit area (which is the 


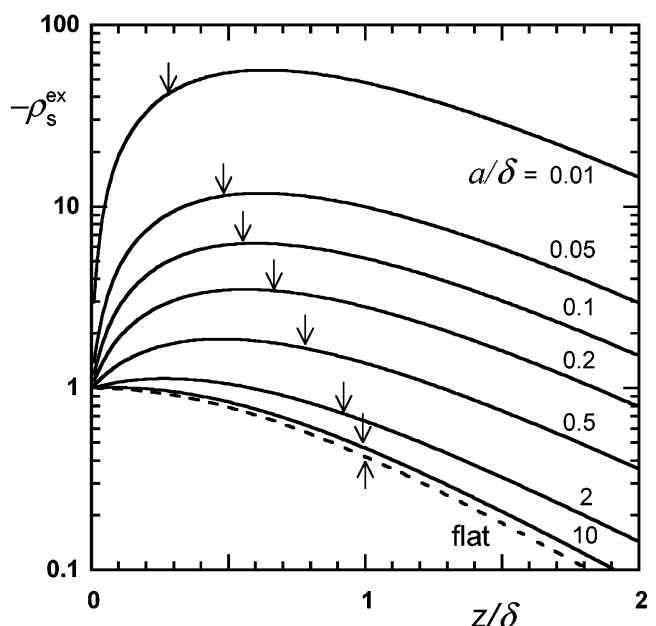

Figure 12. Contribution of a spherical shell at distance $z$ from the sphere surface (eq 36) to the negative adsorption $-\theta_{\mathrm{s}}^{\mathrm{ex}} / \varphi_{\mathrm{b}}$ around a sphere of radius a as a function of the scaled distance $z / \delta$. The ratio a/ $\delta$ is given along each curve; the value of $\delta_{s} / \delta$ is indicated by the arrows. The dashed curve is the "flat" limit $\mathrm{a} / \delta=\infty$

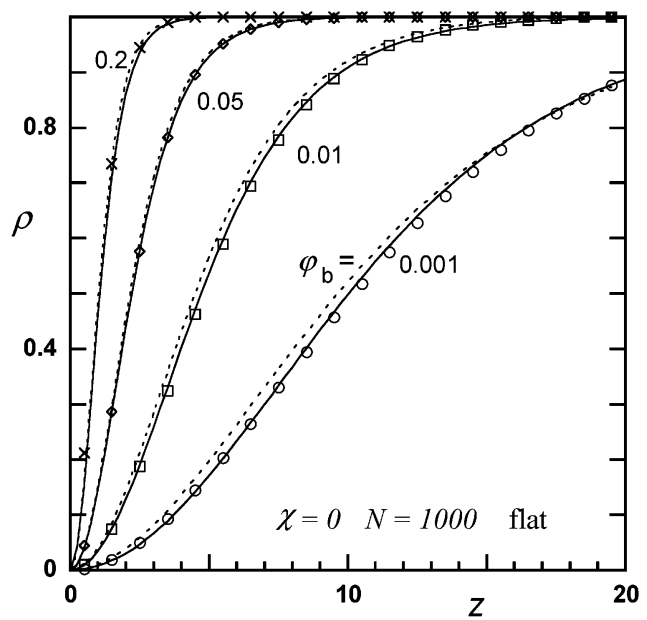

Figure 13. Depletion profiles at a flat plate for four different concentrations (indicated), at $\chi=0$ and $\mathrm{N}=1000$. Solid curves are the analytical result (eq 32), dotted curves are the numerical SS data, and symbols represent SF results.

area under the curves when $\left|\rho_{\mathrm{s}}^{\mathrm{ex}}\right|$ is plotted on a linear scale) strongly increases, due to the second term in eq 36; in the protein limit $\theta_{\mathrm{s}}^{\mathrm{ex}} / \varphi_{\mathrm{b}}$ becomes inversely proportional to a according to eqs 35 and 36 .

8.5. Analytical and Numerical Profiles at a Flat Wall. In the following five figures, we present some representative depletion profiles under various conditions for flat plates (Figures 13 and 14 in this section) and for spheres (Figures 15-17 in section 8.6). From the point of view of our simple theoretical model, they are all special cases of the normalized profiles of $\rho$ as a function of $z / \delta$ for various ratios $a / \delta$, as shown in Figure 11. However, in Figures 13-17 we do not normalize the profiles and plot $\rho(z)$ or $\rho_{s}(z)$ for various conditions (varying $\varphi_{\mathrm{b}}, \chi, \mathrm{N}$, and $\mathrm{a}$ ). This enables a comparison with the predictions of more precise numerical models: that of Schaink-Smit (SS) ${ }^{53}$ (continuum, ground state, no nonlocal effects; we solve eq 13a in ref 53) and the full (discrete) SF model. In all the figures the solid curves are the analytical results, the symbols were computed with the SF model, and the dotted curves represent SS. Because the boundary between the solid

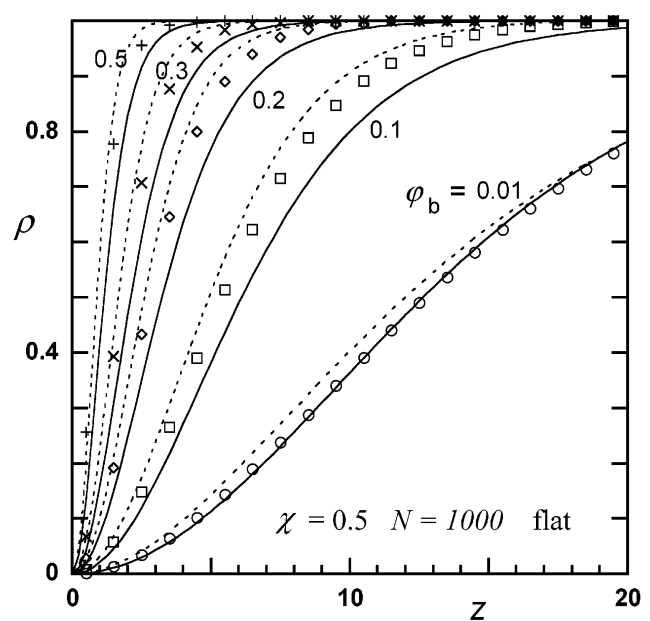

Figure 14. Depletion profiles at a flat plate for five different concentrations (indicated), at $\chi=0.5$ and $\mathrm{N}=1000$. Solid curves are the analytical result (eq 32), dotted curves are the numerical SS data, and symbols represent SF results.

and the liquid is at set at $z=0$, the middle of the layers in the SF profiles shown in Figures $13-17$ is at $z=0.5$, $1.5, \ldots$ (see al so Figure 18).

Figure 13 is for large colloidal particles in a solution of nonadsorbing polymer under good solvency conditions $(\chi=0)$ at different polymer concentrations. The agreement between the analytical model and SF is extremely good, of the same level as shown in Figure 8 for $\delta$, which is the zeroth moment of the profile. Also, SS gives essentially the same result, with only minor deviations.

Figure 14 shows the analogous data set in a $\Theta$ solvent $(\chi=0.5)$. In dilute solutions, as long as $\delta \approx \delta_{0}$, the analytical model describes the profiles excellently, as could be expected from Figure 8, and again SS is very close. When the concentration increases, the analytical model overestimates the width of the profile, as found before (Figure 8). The reason is clear: the approximation $\delta=\xi$ in semidilute $\Theta$ solvents gives an overestimation of order $10 \%$, as discussed in section 5.3 (eq 23 and Figure 5). In these more concentrated solutions, SS is much closer to the SF data, underestimating the profile width slightly. This small difference originates from the neglect of the nonlocal effect in the SS model (see also Figure 5).

8.6. Analytical and Numerical Profiles around a Sphere. Figure 15 demonstrates the effect of the solvency $\chi$ on the profiles around a sphere $(a=20)$, for $\mathrm{N}=1000$ at a concentration $\varphi_{\mathrm{b}}=0.05$, which is in the semidilute range for all solvencies (see Figure 4), with $\xi$ varying from $\xi=2.25$ at $\chi=0$ to $\xi=16.1$ at $\chi=0.5$. In good solvents (up to $\chi \approx 0.4$ ) all three models give essentially the same result. For $\chi=0.47$ and $\chi=0.5$ we see again the overestimation by the analytical model and the slight underestimation by the SS theory. For $\chi$ $=0.5$ the analytical model could be improved by using a modification of eq 32 with $\rho=g^{2}=\tanh ^{2}(\mathrm{z} / \delta)$ replaced by $\rho=\mathrm{g}^{2}=(\mathrm{c}-1) /(\mathrm{c}+2)$, where $\mathrm{c}=\cosh (\sqrt{8 \mathrm{z}} / \delta)$, as in eq 23a. The result (with $\delta$ calculated from eq 27 ) is shown as the dashed curve in Figure 15: it is very close to the SS result, and it underestimates the SF data in a similar way as the "local" sol ution in Figure 5 gives a slightly too narrow profile as compared to the profiles in which the nonlocal term in eq 22 is taken into account.

Figure 16 shows profiles for various chain lengths around a sphere of $\mathrm{a}=20$ at constant concentration $\left(\varphi_{\mathrm{b}}\right.$ 


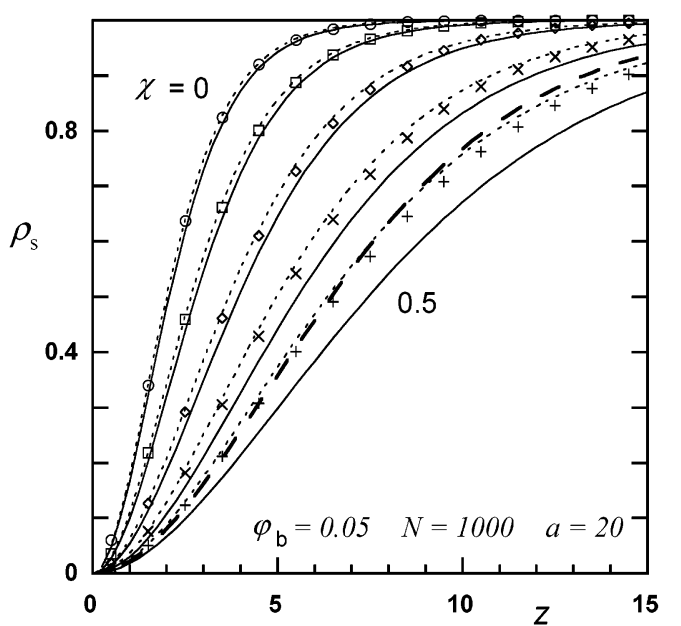

Figure 15. Depletion profiles for $\mathrm{N}=1000$ and $\varphi_{\mathrm{b}}=0.5$ around a sphere of radius $\mathrm{a}=20$ for five different solvencies, with from left to right $\chi=0,0.25,0.4,0.47$, and 0.5 . Solid curves are the analytical result (eq 32), dotted curves are the numerical SS data, and symbols represent SF results. The dashed curve is the "local" $\Theta$ result, as explained in the text.

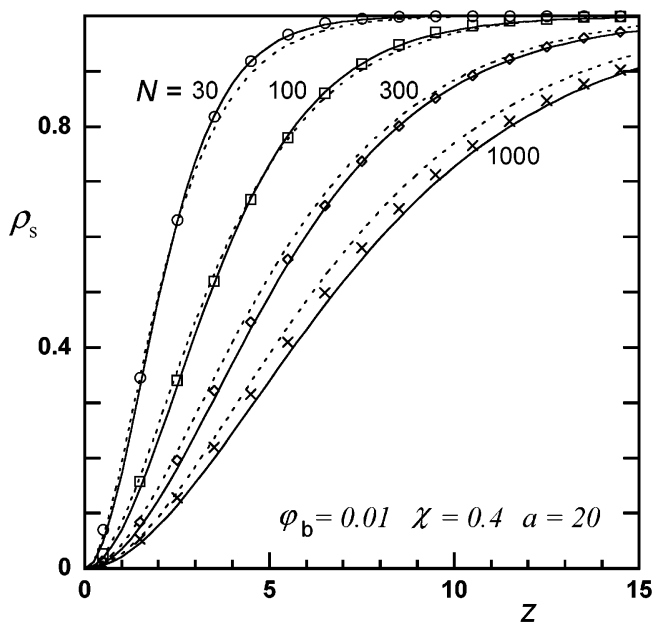

Figure 16. Depletion profiles for $\chi=0.4$ and $\varphi_{\mathrm{b}}=0.01$ around a sphere of radius $a=20$ for four chain lengths (indicated). Solid curves are the analytical result (eq 32), dotted curves are the numerical SS data, and symbols represent SF results.

$=0.01)$ and solvency $(\chi=0.4)$. In all cases the three models give similar results, with the analytical model slightly closer to SF than SS. For high chain lengths SS underestimates the width of the profile somewhat, and the analytical model gives a slight overestimation.

Finally, Figure 17 illustrates, at fixed $\varphi_{\mathrm{b}}(=0.03), \chi$ $(=0.4)$, and $\mathrm{N}(=1000)$, the effect of varying the radius a of the particle. In this case $\left(\delta_{0}=14.6, \delta=6.44\right)$ the particle radius $a=100$ (lowest curve in Figure 17) corresponds to the colloid limit, and all three models give essentially the same profile. As the particles become smaller, the profile becomes narrower because the ratio $\delta_{s} / \delta$ decreases (see Figure 7 ); at the smallest particle size possible in the SF model $(a=1)$, the ratio $a / \delta=$ 0.16 is smaller than unity, but the protein limit is not yet reached. For these small particles, SS is quite close to the SF results, whereas our simple analytical model overestimates the width of the profile slightly. This effect was noted before by Tuinier and Lekkerkerker; ${ }^{43}$ the model could be improved by applying a (rather complicated) correction, as mentioned in section 7.2. We will not apply this correction and are satisfied that our

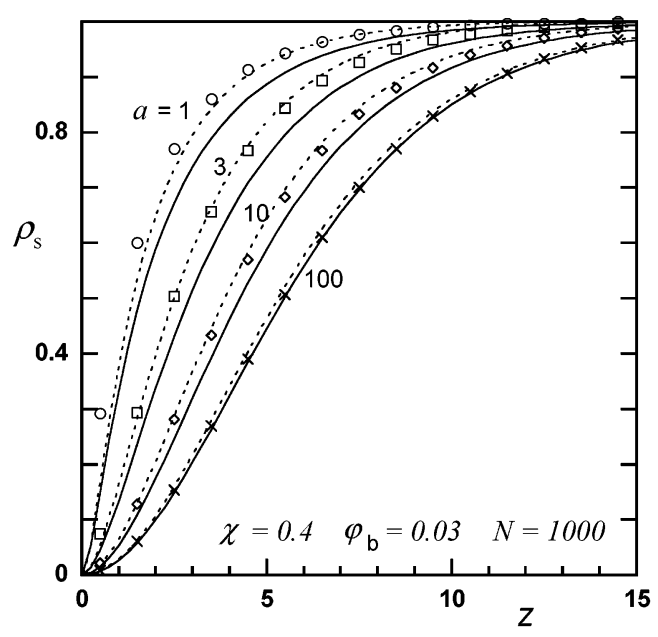

Figure 17. Depletion profiles for $\chi=0.4, \mathrm{~N}=1000$, and $\varphi_{\mathrm{b}}$ $=0.03$ around a sphere, for four particle radii a (indicated). Solid curves are the analytical result (eq 32), dotted curves are the numerical SS data, and symbols represent SF results.

simple, transparent eqs 32 and 27 combined give a quite satisfactory, albeit approximate, description of the trends.

\section{Concluding Remarks}

We have derived a simple, analytical, general continuum expression for the depletion thickness $\delta$ near a flat wall in the mean-field approximation. This equation, $\delta^{-2}=\delta_{0}{ }^{-2}+\xi^{-2}$, is universal in the sense that it covers the entire polymer concentration range for arbitrary solvency and chain length, and it reduces to the known limits $\delta_{0}=2 \mathrm{R} / \sqrt{\pi}$ in dilute solutions and $\delta=\xi$ in the semidilute case. It was derived by using the groundstate approximation (GSA), which gives an approximate, simplified solution of the Edwards equation for polymer trajectories in an external field. The general expression follows from applying GSA to a slit, thereby extending the classical Casassa solution to finite concentrations. The field experienced by the segments in the bulk solution determines the concentration and solvency dependence of the depletion thickness $\delta \approx \xi$ in the semidilute regime. An extended formula for $\xi$ (which is independent of chain length) provides smooth crossovers between various concentration and solvency conditions. The depletion thickness turns out to be equal to a generalized ( $\mathrm{N}$-dependent) bulk solution correlation length.

Both for the dilute and semidilute regimes a simple $\tanh ^{2}(\mathrm{z} / \delta)$ form accurately describes the segment concentration near a flat wall, where $\delta=\delta\left(\varphi_{\mathrm{b}}, \chi, \mathrm{N}\right)$ according to our "universal" expression. The flat-wall results are easily extended to also describe depletion layers around a sphere.

For various conditions, the depletion thickness and the polymer concentration profiles were compared with results of the numerical self-consistent-field (SCF) layer model of Scheutjens and Fleer (SF), which is exact within the mean-field approximation. As to properly compare the analytical continuum model to the discrete SF model, consistent boundary conditions are needed, which are derived in the appendix. Overall, our simple equation is in excellent agreement with the numerical SF results and with a numerical (continuum) GSA model by Schaink and Smit (SS). This demonstrates that GSA is very suitable to describe polymer depl etion. 
The results especially match under conditions of good solvency. For a $\Theta$ solvent at semidilute concentrations, there is a systematic overestimation of about $10 \%$ in the depletion thickness by the analytical model in its simplest form. This is due to the fact that the tanh ${ }^{2}$ profile is not accurate for $\Theta$ conditions. A new analytical result is derived which explains why the deviations occur. In most cases we do not correct for this $10 \%$ so as not to lose the universality.

Our general equation integrates various known results for specific limits (such as the semidilute and dilute (ideal) regimes for a good solvent and a $\Theta$ solvent), and it allows a simple analysis of the effects occurring when the relevant parameters are changed. For example, a quantitative description is given of the well-known effect that the depletion thickness decreases with increasing polymer concentration and that for high polymer concentrations there is no chain-length dependence of the depletion thickness. The polymer concentration at which $\delta$ becomes independent of the chain length decreases for better solvency.

There are also several interesting results on the polymer density and the depletion thickness around a sphere. Whereas the local polymer concentration increases monotonically with increasing radial distance, the excess of polymer in a spherical shell goes through a maximum for small spheres. Moreover, the polymercolloid size ratio $\mathrm{R} / \mathrm{a}$ (or $\delta / \mathrm{a}$ ) influences the depletion thickness $\delta_{s}$ around a sphere. The ratio $\delta_{s} / \delta$ decreases with increasing $\delta / a$, implying that the depletion zone becomes thinner with increasing curvature 1/a. Both increasing the polymer concentration and increasing the polymer-sphere size ratio R/a make the depletion layer narrower.

These consi derations have important implications for the stability of colloids in solutions of nonadsorbing polymer. In a primitive model the depletion-induced attraction is of order $\Pi \vee_{\mathrm{ov}}$, where $\Pi$ is the osmotic pressure of the polymer solution and $\mathrm{V}_{\text {ov }}$ the overlap volume of depletion layers around two spheres. The overlap volume decreases with decreasing $\delta$ and decreasing particle radius. In many cases the trends in $\Pi$ and $V_{\text {ov }}$ are opposite when a variable is changed, so that the overall effect is not obvious. When the polymer concentration is increased, $\Pi$ increases but $V_{\text {ov }}$ decreases, with the steepest decrease around the mi dpoint in Figure 8. When the solvency is made better, again $\Pi$ increases but $V_{\text {ov }}$ decreases. For longer chains $\Pi$ decreases in dilute solutions but $V_{\text {ov }}$ increases. In all these cases the overall effect depends on the relative magnitude of the opposing trends, which can be analyzed with our simple model. Such an analysis will be given in a future paper. The only parameter for which the trends are monotonic is the particle radius: when this is decreased the osmotic pressure is unaffected and $V_{\text {ov }}$ decreases, so that the attraction becomes weaker. Our simple description allows, in principle, a quantitative evaluation of the trends.

We realize that mean-field results have their limitations. Fluctuations are not incorporated, which means that chain expansion, which occurs when the solvency becomes better than the $\Theta$ condition, is absent. Therefore, the mean-field scaling exponents differ from the Flory exponents. For instance, in the semi dilute regime under good solvency conditions mean field gives $\delta \sim$ $\varphi_{\mathrm{b}}{ }^{-1 / 2}$, whereas more accurate treatments accounting for fluctuations predict $\delta \sim \varphi_{\mathrm{b}}{ }^{-3 / 4}$ (Flory) or $\delta \sim \varphi_{\mathrm{b}}{ }^{-0.77}$ (renormalization group theory), which is also found experimentally. Nevertheless, our results provide insight into how the solvent quality, the polymer concentration, and the chain length influence the depletion thickness. There are analytical and computer simulation results both for polymer chains in the full excludedvolume limit with fluctuations and for ideal chains, which can be used for comparison and to estimate the quantitative errors. We have some indi cations that our simple model can be adapted such as to accurately describe Monte Carlo (excluded-volume) results for the distribution coefficient in size-exclusion chromatography.

In the concentrated regime, where no other analytical results are available so far, the mean-field description is accurate. Fluctuations are suppressed when approaching polymer melt conditions, where the depletion thickness becomes very small as follows from our simple equation.

We have made an extension from the flat wall to spherical geometry. It may al so be interesting to extend the description toward other geometries such as a cylinder. This may help to describe the depletion of polymer chains around a colloidal rod or a virus.

Acknowledgment. This research was made possible by INTAS Grant 2000-0031 and by the NWO DutchRussian program "Self-organisation and structure of bionanocomposites" which enabled A.M.S. to visit Wageningen. We thank Dr. Frans Leermakers for help with the SF computations, Prof. Erich Eisenriegler for useful remarks on the manuscript, and Dr. Henny Schaink for making his SS program available to us.

\section{Appendix. Boundary Condition in Continuum and Lattice Models}

In this paper we have demonstrated that a good approximation for the depletion profile is $\rho=g^{2}=\tanh ^{2}$ $(z / \delta)$. This form is based upon the boundary condition $g(0)=0$ at $z=0$, the interface between the solid and the liquid phase. This boundary condition implies a certain choice for the segment/surface repulsion in the lattice model, as we show in this appendix. We discuss this boundary condition both for the continuum model (where all space can be used up to the interface at $z=$ 0 ) and for a lattice model (where the center of a segment has to stay away half a bond length from the solid). In a recent paper, the mapping of continuum and lattice models for the case of a single end-attached chain was discussed, ${ }^{57}$ and Van der Gucht et al. ${ }^{58}$ did the same for a dilute polymer solution in contact with a surface close to the adsorption/desorption transition.

The segment/wall repulsion may be characterized by the Sil berberg adsorption energy parameter $\chi_{s},{ }^{59}$ which is below the critical adsorption energy $\chi_{\mathrm{sc}}$ in the case of depletion. For a repulsive surface it is negative; for strong repulsion $\chi_{\mathrm{s}}=-\infty$. This parameter can also be expressed as $\chi_{\mathrm{s}}=-\chi_{\mathrm{PS}} / 6$ for a six-choice cubic lattice, ${ }^{31,60}$ where $\chi_{\text {PS }}$ is the Flory-Huggins interaction parameter between polymer segments and "segments" of the solid. It is useful to define $\Delta \chi \equiv \chi_{\mathrm{PS}}-\chi$, which measures the difference in contact energy between the combination segment/solid and the pair segment/solvent. Clearly, for a strongly repulsive surface $\Delta \chi=\infty$. When $\Delta \chi=0$, we have a neutral surface in the sense that the solid has the same contact energy with a polymer segment as the solvent, so that the presence of the surface has only 


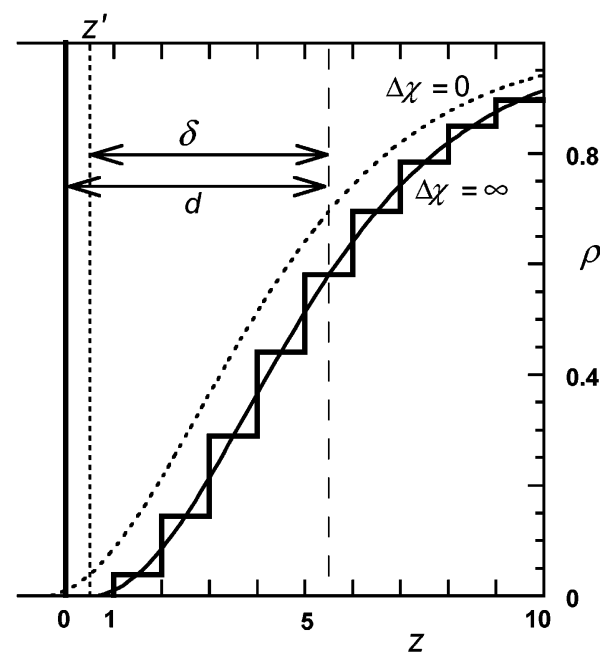

Figure 18. Continuum and discrete profiles for a strongly repulsive surface ( $\Delta \chi=\infty$, solid curve/steps) and the continuous profile for a neutral surface $(\Delta \chi=0$, dotted). The continuous profiles start at $z=z^{\prime}$, where $z^{\prime}=0.5$ (vertical dotted line) for $\Delta \chi=\infty$ and $z^{\prime}=-0.5$ for $\Delta \chi=0$. The width $\delta$ of the profiles is $\delta=5$ in both cases; the vertical dashed line at $z=5.5$ intersects the profile at $\rho=\tanh ^{2} 1=0.58$. The depletion thickness $\mathrm{d}$ equals $\delta+z^{\prime}$, which is 5.5 for $\Delta \chi=\infty$ (as indicated) and 4.5 for $\Delta \chi=0$ (not shown).

consequences for the entropy of the polymer chains, and not for their energy.

When a continuous function $\rho(z)$ is used to describe lattice data in discretized space with layers $i=0,1,2$, ... (where $i$ is an integer and layer 0 belongs to the solid), an obvious choice is to assign the continuum value in the middle of each layer $\mathrm{i}$, at position $\mathrm{z}_{\mathrm{i}}=\mathrm{i}-0.5$, to the entire layer. In the depletion case we consider the tanh $^{2}$ profile, but when we fit such a profile through lattice data, in general the $\tanh ^{2}$ is not zero at $z=0$ but at some position $z=z^{\prime}$ which is close to (but not equal to) zero: for arbitrary $\Delta \chi$ the continuum boundary condition is then $\rho\left(z^{\prime}\right)=0$. The continuous and discrete versions of the profile are

$$
\begin{gathered}
\rho(z)=\tanh ^{2}\left(\frac{z-z^{\prime}}{\delta}\right) \\
\rho_{\mathrm{i}}=\tanh ^{2}\left(\frac{z_{i}-z^{\prime}}{\delta}\right)
\end{gathered}
$$

where $\delta$ is again the width (zeroth moment) of the profile (from $z=z^{\prime}$ ) and is still given by eq 27 . It is thus equal to $-\theta^{\prime} / \varphi_{\mathrm{b}}$, where $\theta^{\prime}$ is the excess amount for the region $z>z^{\prime}$. The depletion thickness, which we denote by $\mathrm{d}$, is defined by $\mathrm{d}=-\theta^{\mathrm{ex}} / \varphi_{\mathrm{b}}$, with $\theta^{\mathrm{ex}}$ the excess amount for the region $z^{\prime}>0$. Hence, the width $\delta$ of the profile is only equal to the depletion thickness $d$ when $z^{\prime}=0$, which corresponds to the boundary condition $\rho$ $(0)=0$ as used in the main text.

The value of $z^{\prime}$ depends on $\Delta \chi$. An example for a strongly repulsive surface $(\Delta \chi=\infty)$ is given in Figure 18. In this case the first layer $(i=1)$ is empty: $\rho_{1}=0$, which with eq $A l b$ leads to $z^{\prime}=z_{1}=0.5$ and to $a$ continuum boundary condition $\rho(0.5)=0$ according to eq Ala. The solid curve in Figure 18 is the continuous profile, and the discrete profile is also indicated. This situation corresponds to a potential profile $u_{i}=\infty$ for $\mathrm{i}$ $\leq 1$ (solid plus first sol ution layer) and $\mathrm{u}_{\mathrm{i}} \approx 0$ for $\mathrm{i}=2$. We see from Figure 18 that $\mathrm{d}=\delta+0.5$ for $\Delta \chi=\infty: \delta$ is the area above the solid curve up to $z^{\prime}=0.5$ (dotted vertical line), and $d$ is the area up to $z=0$ (solid vertical line).

When we consider a neutral surface $(\Delta \chi=0)$, the potential profile shifts one unit to the left: $u_{i}=\infty$ for $\mathrm{i}$ $\leq 0$ (within the solid) and $u_{i} \approx 0$ for $\mathrm{i}=1$. Now $\rho_{0}=0$ and $z^{\prime}=z_{0}=-0.5$, with boundary condition $\rho(-0.5)=$ 0 in the continuum model. The shift over one bond length is easily visualized: upon making a neutral surface $(\Delta \chi=0)$ strongly repulsive $(\Delta \chi=\infty)$, the only effect is that the first layer is made inaccessible, and a new neutral interface is formed at the boundary between the first and second layers.

It follows from Figure 18 that there is a simple relation between the depletion thickness $d$ and the width $\delta$ of the $\tanh ^{2}$ profile:

$$
\mathrm{d}=\delta+\mathrm{z}^{\prime}
$$

In the continuum model discussed in this paper we have used the special case $z^{\prime}=0, \delta=d$ because of the imposed boundary condition $\mathrm{g}(0)=0$. This must correspond to a value of $\Delta \chi$ in between the two limits shown in Figure 18. This value may be found from the discrete boundary condition.

The discrete form of the Edwards equation is the recurrence relation $\left.G_{i, s+1}=e^{u_{b}-u_{i}\left\langle G_{i, s}\right.}\right\rangle$, where $\left\langle G_{i, s}\right\rangle \equiv$ $\left[\mathrm{G}_{\mathrm{i}-1, \mathrm{~s}}+4 \mathrm{G}_{\mathrm{i}, \mathrm{s}}+\mathrm{G}_{\mathrm{i}+1, \mathrm{~s}}\right] / 6$. The boundary condition is $\mathrm{G}_{0, \mathrm{~s}}$ $=0$ and a given value of $\Delta \chi$ entering the potential $u_{1}$ in the surface layer. Hence, for $\mathrm{i}=1$ and $\mathrm{s}=\mathrm{N}$

$$
\begin{gathered}
{\left[4 \mathrm{G}_{1, \mathrm{~N}}+\mathrm{G}_{2, \mathrm{~N}}\right] / \mathrm{G}_{1, \mathrm{~N}+1}=6 \mathrm{e}^{\mathrm{u}_{1}-\mathrm{u}_{\mathrm{b}}}} \\
\mathrm{u}_{1}=-\ln \left(1-\varphi_{1}\right)-2 \chi\left\langle\varphi_{1}\right\rangle+\Delta \chi / 6
\end{gathered}
$$

We first consider the case of small $\varphi_{\mathrm{b}}$ (and even smaller $\left.\varphi_{1}\right)$. Then $\mathrm{u}_{1}-\mathrm{u}_{\mathrm{b}} \approx \Delta \chi / 6$. In the semidilute limit we have $G_{i, N} \approx g_{i}=\tanh \left[\left(z_{i}-z^{\prime}\right) / \delta\right]$, which reduces to $\left(z_{i}-\right.$ $\left.z^{\prime}\right) / \delta$ for $i=1,2$. In the dilute limit we have $G_{i, N} \approx$ erf$\left[\left(z_{i}-z^{\prime}\right) / 2 \sqrt{N / 6}\right]$ according to eq $6 a$, which reduces to $\sqrt{6 / \pi}\left(z_{i}-z^{\prime}\right) / \sqrt{N}$ for $i=1,2$. When we approximate $\sqrt{N}$ $\approx \sqrt{\mathrm{N}+1}$, we find in both cases for the left-hand side of eq A3a $4+\left(1.5-z^{\prime}\right) /\left(0.5-z^{\prime}\right)$. Hence the relation between $\Delta \chi$ and $z^{\prime}$ is

$$
\begin{gathered}
\frac{\Delta \chi}{6}=\ln \left(\frac{7-10 z^{\prime}}{6-12 z^{\prime}}\right) \\
2 z^{\prime}=\frac{e^{\Delta \chi / 6}-7 / 6}{e^{\Delta x / 6}-5 / 6}
\end{gathered}
$$

Equation A4b corroborates that $z^{\prime}=-0.5$ and $d=\delta-$ 0.5 for a neutral surface $(\Delta \chi=0)$ and that $z^{\prime}=0.5$ and $\mathrm{d}=\delta+0.5$ for a strongly repulsive surface $(\Delta \chi=\infty)$. The latter case is indicated in Figure 18. Equation A4a enables us to find which value of $\Delta \chi$ corresponds to $z^{\prime}$ $=0$, according to the boundary condition $\mathrm{g}(0)=0$ as used in the main paper: $\Delta \chi=6 \ln (7 / 6) \approx 1$. This is the value used for all SF results presented in this paper; it is adequate for $\varphi_{\mathrm{b}}$ bel ow $\sim 0.15$. We have checked from numerical SF calculations that in not too concentrated solutions $\mathrm{d}=-\theta^{\mathrm{ex}} / \varphi_{\mathrm{b}}=\sum_{\mathrm{i}}\left(1-\rho_{\mathrm{i}}\right)$ is increased by 0.5 when the repulsion is increased from $\Delta \chi=0$ to $\Delta \chi=1$ and again by 0.5 when a very large value of $\Delta \chi$ is taken.

In concentrated solutions, eq A4 is not accurate enough. When $\varphi_{\mathrm{b}}>0.15$, we have to retain the concentration terms in eq A3. When we approximate $\rho_{\mathrm{i}}$ by eq Alb and $g_{\mathrm{i}} \approx \sqrt{\rho_{\mathrm{i}}}$, we find 


$$
\begin{array}{r}
\frac{\Delta \chi}{6}=\ln \left(\frac{4}{6}+\frac{1}{6} \frac{g_{2}}{g_{1}}\right)+\ln \left(\frac{1-\varphi_{b} g_{1}^{2}}{1-\varphi_{b}}\right)+ \\
\frac{\chi \varphi_{b}}{3}\left(4 g_{1}^{2}+g_{2}^{2}-6\right)
\end{array}
$$

where $g_{1}=\tanh \left[\left(0.5-z^{\prime}\right) / \delta\right]$ and $g_{2}=\tanh \left[\left(1.5-z^{\prime}\right) /\right.$ $\delta$ ], with $\delta=\delta\left(\varphi_{\mathrm{b},}, \chi_{,} \mathrm{N}\right)$ according to eq 27 . For small $\varphi_{\mathrm{b}}$ only the first term remains, and with $g_{2} / g_{1} \approx\left(1.5-z^{\prime}\right) /$ $\left(0.5-z^{\prime}\right)$ eq $A 5$ reduces to eq A4a. Then the value of $\Delta \chi$ for which $z^{\prime}=0$ is a constant $(\Delta \chi \approx 1)$. For higher $\varphi_{\mathrm{b}}$ the value of $\Delta \chi$ which corresponds to $g(0)=0$ is found by substituting $z^{\prime}=0$ in $g_{1}$ and $g_{2} ; \Delta \chi$ becomes now a function of concentration, solvency, and chain length and is usually higher than 1 . It is also possible to calculate the value of $z^{\prime}$ to be used in the continuum model to describe a lattice calculation for arbitrary $\Delta \chi$ (i.e., a value for which $g\left(z^{\prime}\right)=0$ with $z^{\prime} \neq 0$ ); then eq A5 has to be solved numerically for $z^{\prime}$, at given values of $\Delta \chi$ and $\delta=\delta\left(\varphi_{\mathrm{b},}, \mathrm{N}\right)$, and eq Ala with $\mathrm{g}\left(\mathrm{z}^{\prime}\right)=0$ and $\mathrm{z}^{\prime}$ $\neq 0$ has to be used for the concentration profile.

\section{References and Notes}

(1) Poon, W. C. K. J . Phys.: Condens. Matter 2002, 14, R859.

(2) Doublier, J .-L.; Garnier, C.; Renard, C.; Sanchez, C. Curr. Opin. Colloid Interface Sci. 2000, 5, 184.

(3) Hughes, D. F. K.; Robb, I. D.; Dowsing, P. J . Langmuir 1999, $15,5227$.

(4) Snyder, L. R.; Kirkland, J . J . Introduction to Modern Liquid Chromatography; Wiley-I nterscience: New York, 1979.

(5) Asakura, S.; Oosawa, F. J . Chem. Phys. 1954, 22, 1255.

(6) Asakura, S.; Oosawa, F. J . Polym. Sci. 1958, 33, 183.

(7) Vrij, A. Pure Appl. Chem. 1976, 48, 471.

(8) De Hek, H.; Vrij, A. J . Colloid Interface Sci. 1981, 84, 409.

(9) Gast, A. P.; Hall, C. K.; Russel, W. B. J . Colloid Interface Sci. 1983, 96, 251.

(10) Lekkerkerker, H. N. W.; Poon, W. C. K.; Pusey, P. N.; Stroobants, A.; Warren, P. B. Europhys. Lett. 1992, 20, 559.

(11) Ilett, S. M.; Orrock, A.; Poon, W. C. K.; Pusey, P. N. Phys. Rev. E 1995, 51, 1344.

(12) Taniguchi, T.; Kawakatsu, T.; Kawasaki, K. In Slow Dynamics in Condensed Matter; Kawasaki, K., Kawakatsu, T., Tokuyama, M., Eds.; AIP Conference Proceedings Vol. 256; American Institute of Physics: New York, 1992; p 503.

(13) Eisenriegler, E.; Hanke, A.; Dietrich, S. Phys. Rev. E 1996, 54, 1134

(14) Tuinier, R.; Vliegenthart, G. A.; Lekkerkerker, H. N. W. J . Chem. Phys. 2000, 113, 10768.

(15) Meijer, E. J .; Frenkel, D. J . Chem. Phys. 1994, 100, 6873.

(16) J oanny, J . F.; Leibler, L.; De Gennes, P. G. J . Polym. Sci., Polym. Phys. 1979, 17, 1073.

(17) Maassen, R.; Eisenriegler, E.; Bringer, A. J . Chem. Phys. 2001, 115, 5292.

(18) Fuchs, M.; Schweizer, K. S. Europhys. Lett. 2000, 51, 621; Phys. Rev. E 2001, 64, 021514.

(19) Tuinier, R.; Lekkerkerker, H. N. W. Eur. Phys. J . E 2001, 6, 129.

(20) Bolhuis, P. G.; Louis, A. A.; Hansen, J . P.; Meijer, E. J . J . Chem. Phys. 2001, 114, 4296.
(21) Hanke, A.; Eisenriegler, E.; Dietrich, S. Phys. Rev. E 1999, $59,6853$.

(22) Schlesener, F.; Hanke, A.; Klimpel, R.; Dietrich, S. Phys. Rev. E 2001, 63, 041803.

(23) Klos, J .; Pakula, T. J . Chem. Phys. 2003, 118, 1507.

(24) Milchev, A.; Binder, K. Eur. Phys. J . B 1998, 3, 477.

(25) Aarts, D. G. A. L.; Tuinier, R.; Lekkerkerker, H. N. W. J . Phys.: Condens. Matter 2002, 14, 7551.

(26) Bolhuis, P. G.; Louis, A. A.; Hansen, J .-P. Phys. Rev. Lett. 2002, 89, 128302

(27) Schmidt, M.; Fuchs, M. J . Chem. Phys. 2002, 117, 6308.

(28) Schmidt, M.; Denton, A. R.; Brader, J . M. J . Chem. Phys. 2003, 118, 1541.

(29) Scheutjens, J. M. H. M.; Fleer, G. J . J . Phys. Chem. 1979, 83, 1619.

(30) Scheutjens, J . M. H. M.; Fleer, G. J . Adv. Colloid Interface Sci. 1982, 16, 361.

(31) Fleer, G. J .; Cohen Stuart, M. A.; Scheutjens, J . M. H. M.; Cosgrove, T.; Vincent, B. Polymers at Interfaces; Chapman $\&$ Hall: London, 1993.

(32) Van der Gucht, J .; Besseling, N. A. M.; Van Male, J .; Cohen Stuart, M. A. J . Chem. Phys. 2000, 112, 2886.

(33) De Gennes, P. G. Macromolecules 1981, 14, 1637.

(34) Louis, A. A.; Bolhuis, P. G.; Meijer, E. J .; Hansen, J .-P. J . Chem. Phys. 2002, 116, 10547

(35) Louis, A. A.; Bolhuis, P. G.; Meijer, E. J .; Hansen, J .-P. J Chem. Phys. 2002, 117, 1893.

(36) Tuinier, R.; Lekkerkerker, H. N. W.; Aarts, D. G. A. L. Phys. Rev. E 2002, 65, R060801.

(37) De Gennes, P. G. C. R. Acad. Sci. B 1980, 290, 509

(38) De Gennes, P. G. Scaling Concepts in Polymer Physics; Cornell University Press: Ithaca, NY, 1979.

(39) Edwards, S. F.; Freed, K. F. J . Phys. A 1969, 2, 145

(40) Doi, M.; Edwards, S. F. The Theory of Polymer Dynamics; Clarendon Press: Oxford, 1986.

(41) Grosberg, A. Y.; Khoklov, A. R. Statistical Mechanics of Macromolecules; AIP: New York, 1994.

(42) Odijk, T. Macromolecules 1996, 29, 1842.

(43) Tuinier, R.; Lekkerkerker, H. N. W. Macromolecules 2002, 35, 3312.

(44) Huggins, M. L. J . Chem. Phys. 1941, 9, 440.

(45) Flory, P. J .J . Chem. Phys. 1941, 9, 660; Principles of Polymer Chemistry; Cornell University Press: Ithaca, NY, 1953.

(46) Helfand, E. J . Phys. Chem. 1975, 62, 999.

(47) Hong, K. M.; Noolandi, J. Macromolecules 1981, 14, 727.

(48) Eisenriegler, E.J. Chem. Phys. 1983, 79, 1052.

(49) Eisenriegler, E. Polymers near Surfaces; World Scientific: Singapore, 1993.

(50) Eisenriegler, E.; Maassen, R. J . Chem. Phys. 2002, 116, 449

(51) Casassa, E. F. J . Polym. Sci., Part B 1967, 5, 773. Casassa, E. F.; Tagami, Y. Macromolecules 1969, 2, 14.

(52) Fleer, G. J .; Van Male, J .; J ohner, A. Macromol ecules 1999, $32,825,845$.

(53) Schaink, H. M.; Smit, J . M. J . Chem. Phys. 1997, 107, 1004.

(54) DiMarzio, E. A.; Rubin, R. J . J . Chem. Phys. 1971, 55, 4318.

(55) Eisenriegler, E. J . Chem. Phys. 2000, 113, 5091.

(56) Eisenriegler, E., private communication.

(57) Gorbunov, A. A.; Skvortsov, A. M.; Fleer, G. J . J . Chem. Phys. 2001, 114, 5366.

(58) Van der Gucht, J .; Besseling, N. A. M.; Fleer, G. J . Macromolecules 2002, 35, 2810.

(59) Silberberg, A. J . Chem. Phys. 1968, 48, 2835.

(60) Evers, O. A.; Scheutjens, J . M. H. M.; Fleer, G. J . Macromolecules 1990, 23, 5221.

MA0345145 